\title{
Modeling velocity autocorrelation functions of confined fluids: A memory function approach
}

\author{
S. H. Krishnan and K. G. Ayappa ${ }^{a}$ \\ Department of Chemical Engineering, Indian Institute of Science, Bangalore-India 560012
}

\begin{abstract}
Velocity autocorrelation functions (VACF) of a fluid confined in a slit pore have been modeled using the memory equation. Models for the VACF are based on both the truncation and analytic closure approximations of the Mori's continued fraction representation. The performance of the models is evaluated for gas to liquid-like pore densities and pore widths which accommodate one to four atomic layers. In all cases we compare the predictions from the models with the VACF obtained from molecular dynamics simulations. The truncation models predict an oscillatory behavior for the in-plane VACF with better agreement at lower densities. Among the analytical closure models we observe that the sech model applied at the first level of closure is not only able to capture the short-time dynamics but is also seen to give the best predictions to the in-plane diffusivities at liquid-like pore densities. Although the minima in the VACFs are captured accurately by the sech model, the subsequent plateau regions in the VACF typically observed in confined systems are not predicted. This aspect is due to the slower relaxation of the actual memory kernel, which is not captured by the model. Predictions of the in-plane diffusivities using different levels of analytic closure have been compared with diffusivities obtained from the simulations.
\end{abstract}

\section{INTRODUCTION}

Fluids confined in molecular dimensions between solid surfaces are encountered in a variety of processes such as adsorption, wetting, boundary lubrication, and heterogeneous catalysis. Such systems are observed in diverse applications such as chromatography, oil recovery, and membrane separation technologies. Due to the strong interactions of the fluid molecules with the confining walls, the fluid is inhomogeneous and the spatial distribution of particles is known to influence the static and dynamic properties of the confined fluid. ${ }^{1,2}$

Molecular dynamics (MD) simulations have been extensively used to study transport properties of confined fluids. In particular, the self-diffusivities of single component fluids confined in slit-shaped nanopores have been widely investigated. ${ }^{3-6}$ The variation of the diffusion coefficients on the density, layering, and state of the pore fluid is now well documented. ${ }^{7}$ Although molecular dynamics simulations have been widely used to study the transport properties of confined fluids, theoretical treatments of the transport properties of the confined inhomogeneous fluid are relatively few.

A theory for the self-diffusion coefficients of inhomogeneous fluids based on the Enskog kinetic theory for dense gases has been developed for confined fluids. ${ }^{8}$ When compared with the self-diffusivity values obtained from MD simulations, the theory is able to qualitatively predict the variations in diffusion coefficients as a function of pore width for smooth fluid-wall potentials. ${ }^{9,10}$ However the theory is seen to underestimate diffusivities for structured pores. ${ }^{10}$ Other kinetic theory-based approaches ${ }^{11-15}$ which

${ }^{a)}$ Electronic mail: ayappa@chemeng.iisc.ernet.in seek to provide a description for transport properties of strongly inhomogeneous fluids are based on kinetic theories developed for homogeneous fluids. ${ }^{16}$ Although kinetic theory-based approaches for bulk fluids have been extended to develop a theory for transport properties of highly inhomogeneous fluids, liquid state theories based on time correlation functions have not been applied to confined fluids.

Within the framework of equilibrium statistical mechanics, the transport properties of a fluid are related to the time correlation functions of the relevant dynamic variables via appropriate Green-Kubo relations. ${ }^{17}$ The velocity autocorrelation functions (VACF) are used to determine the selfdiffusivity of a fluid. If the appropriate time correlation functions can be predicted, then the relevant transport properties can in principle be determined. In the memory function approach the autocorrelation of any dynamic variable is related to its time derivative through a convolution with a memory kernel. ${ }^{18}$ The success of using the memory function formalism relies on obtaining a suitable form for the memory kernel. In this regard many different approaches have been used. These include kinetic, hydrodynamic, and mode-coupling theories. ${ }^{18,19}$ A classical approach is the Mori continued fraction procedure, which reduces to determining a hierarchy of memory functions. ${ }^{18}$ Various levels of truncation of the continued fraction representation give rise to different levels of approximation of the autocorrelation function. This approach has been widely used in modeling the velocity autocorrelation function for a bulk fluid, and also has the advantage that it can be systematically improved. It involves evaluating the moments of the power spectrum of the VACF, which are also known as the Mori constants.

An alternative approach is heuristic in nature and relies 
on proposing an appropriate form of the memory kernel whose constants are related to the moments of the power spectrum of the VACF. Alternatively, this procedure can be viewed as a process of analytic closure ${ }^{20,21}$ for the hierarchy of memory functions that arises in a continued fraction representation. In this regard the sech closure ${ }^{22-25}$ of the memory kernel is able to predict self-diffusivities obtained from molecular dynamics data over a wide range of densities and temperatures. ${ }^{20,23}$ Recently, methods from signal processing have been used to obtain the memory function by assuming that the dynamical process is governed by an underlying stochastic process. ${ }^{26}$

In this work we apply the memory function formalism that has been developed for bulk fluids to a fluid confined in a slit nanopore. In all cases the predicted VACF was compared with the VACF obtained from MD simulations. Six models which have been successful in capturing most of the features of the VACF of a bulk fluid have been considered. Two of them emerge out of the Mori continued fraction procedure at the second and third level truncations, and the rest are analytic closure-based models for the memory function. The Mori constants which are used as inputs into the models are obtained directly from MD simulation. Simulations of the confined fluid were carried out in the canonical ensemble $(N V T)$ using constrained dynamics. ${ }^{27-30}$ Simulations were carried out for different pore widths which accommodate one to four fluid layers. As the physics of confined fluids can depend on the molecular nature of the confining walls, results were compared for pores with molecularly structured and smooth walls. Since the fluid molecules experience an unbalanced force in the direction parallel to walls in a structured pore, the total momentum of the fluid is not a conserved quantity. Systematic system size checks were carried out to ascertain the magnitude of this effect.

\section{THEORY}

\section{A. The memory equation}

Within the framework of equilibrium statistical mechanics, autocorrelation functions form the starting point for the study of transport coefficients of fluids. Appropriate GreenKubo formulas relate the long-time integrals of autocorrelation functions to a given transport property. Thus, the selfdiffusivity $D$ is related to the VACF in the following manner:

$$
D=\frac{k_{B} T}{m} \int_{0}^{\infty} \Psi(t) d t,
$$

where $k_{B}$ is the Boltzmann constant, $T$ the temperature, and $m$ mass of the diffusing particle. In Eq. (1) the normalized velocity autocorrelation function

$$
\Psi(t)=\frac{\langle\mathbf{v}(0) \cdot \mathbf{v}(t)\rangle}{\left\langle|\mathbf{v}(0)|^{2}\right\rangle},
$$

where $\left\langle|\mathbf{v}(0)|^{2}\right\rangle=\left(3 k_{B} T / m\right)$. The objective of the memory function approach is to provide a theoretical framework to predict the time evolution of the VACF. The dynamical equation for the VACF is a first kind Volterra integro differential operator $^{18}$ given by

$$
\dot{\Psi}(t)=-\int_{0}^{t} K_{1}\left(t-t^{\prime}\right) \Psi\left(t^{\prime}\right) d t^{\prime},
$$

where the time derivative of the VACF, $\dot{\Psi}(t)$, is expressed as a convolution of the memory function $K_{1}(t)$ and the VACF. Equation (2), which is referred to as the memory equation (ME) or the master equation, can be formally derived from the Liouville equation with an application of the projection operator formalism. ${ }^{18}$ In this development, the memory function, expressed in terms of projection operator $\mathcal{P}$ and Liouville operator $\mathcal{L}$ is

$$
K_{1}(t)=\frac{\left\langle\dot{v}(0)(1-\mathcal{P}) e^{(1-\mathcal{P}) \mathcal{L} t} \dot{v}(0)\right\rangle}{\left\langle|v(0)|^{2}\right\rangle} .
$$

Since the projection operator approach is developed for the time evolution of any dynamical quantity, a hierarchy of memory functions $K_{n}(t), n>0$ can be shown to satisfy the memory equation Eq. (2). Hence

$$
\dot{K}_{n}(t)=-\int_{0}^{t} K_{n+1}\left(t-t^{\prime}\right) K_{n}\left(t^{\prime}\right) d t^{\prime} .
$$

For $n=0, K_{0}(t)=\Psi(t)$ and Eq. (4) reduces to the ME, Eq. (2). In order to derive the continued fraction representation of $\Psi(t)$, one begins by taking Laplace transforms of Eq. (4), which results in

$$
s \widetilde{K}_{n}(s)-K_{n}(0)=-\widetilde{K}_{n}(s) \widetilde{K}_{n+1}(s),
$$

where the Laplace transform

$$
\widetilde{K}_{n}(s)=\int_{0}^{\infty} K_{n}(t) e^{-s t} d t .
$$

Equation (5) can be rewritten as

$$
\widetilde{K}_{n}(s)=\frac{K_{n}(0)}{s+\widetilde{K}_{n+1}(s)}, \quad n=0,1,2, \ldots .
$$

Noting that $\widetilde{K}_{0}(s)=\widetilde{\Psi}(s)$, Eq. (5) can be used to derive the continued fraction representation for $\widetilde{\Psi}(s)$

$$
\widetilde{\Psi}(s)=\frac{K_{0}(0)}{s+\frac{K_{1}(0)}{s+\frac{\ldots K_{n}(0)}{s+\widetilde{K}_{n+1}(s)}}},
$$

where $K_{n}(0)$ are referred to as Mori constants.

We next relate the Mori constants $\left[K_{n}(0)\right]$ to the frequency moments of the VACF. Noting that $\Psi(t)$ is an even function of time, the Taylor series representation (relating the even powers of $t$ ) around $t=0$

$$
\Psi(t)=1-\frac{\omega_{v}^{2} t^{2}}{2 !}+\frac{\omega_{v}^{4} t^{4}}{4 !}+\cdots,
$$

where the frequency moments

$$
\omega_{v}^{2 n}=(-1)^{n}\left(\frac{d^{2 n} \Psi}{d t^{2 n}}\right)_{t=0}, \quad n=1,2, \ldots .
$$

Noting that the Fourier transform pairs are 


$$
\Psi(\omega)=\int_{0}^{\infty} e^{-i \omega t} \Psi(t) d t
$$

and

$$
\Psi(t)=\frac{1}{2 \pi} \int_{0}^{\infty} e^{i \omega t} \Psi(\omega) d \omega,
$$

Eqs. (10) and (12) can be used obtain the following expression for the frequency moments:

$$
\omega_{v}^{2 n}=\frac{1}{2 \pi} \int_{0}^{\infty} \omega^{2 n} \Psi(\omega) d \omega .
$$

In order to relate the Mori constants $K_{n}(0)$ to the moments $\omega_{v}^{2 n}$, we take the Laplace transform of Eq. (9) to obtain

$$
\tilde{\Psi}(s)=\frac{1}{s}-\frac{\omega_{v}^{2}}{s^{3}}+\frac{\omega_{v}^{4}}{s^{5}}-\cdots .
$$

Comparing Eq. (14) and Eq. (8) at different levels of truncation yields

$$
K_{0}(0)=1, \quad K_{1}(0)=\omega_{v}^{2}, \quad K_{2}(0)=\left(\frac{\omega_{v}^{4}}{\omega_{v}^{2}}\right)-\omega_{v}^{2},
$$

and

$$
K_{3}(0)=\frac{\left(\frac{\omega_{v}^{6}}{\omega_{v}^{4}}-\frac{\omega_{v}^{4}}{\omega_{v}^{2}}\right)}{\left(1-\frac{\left(\omega_{v}^{2}\right)^{2}}{\omega_{v}^{4}}\right)} .
$$

An alternative way of obtaining the results of Eq. (15) is by taking a time derivative of Eq. (2) and evaluating the result at time $t=0$. Using this procedure

$$
K_{1}(0)=-\frac{\left\langle\dot{v}(0)^{2}\right\rangle}{\left\langle v(0)^{2}\right\rangle} .
$$

Higher time derivatives of Eq. (2) can be used to relate the initial values of the memory kernels, $K_{n}(0), n>1$, to the higher order frequency moments of $\Psi(t)$, recovering the relations given in Eq. (15).

\section{B. Truncation schemes: The second- and third-order truncation models}

In the truncation method the continued fraction representation is truncated at the $n$th level by retaining terms in the fraction up to $\widetilde{K}_{n}(s)$ and replacing $\widetilde{K}_{n}(s)$ by $\widetilde{K}_{n}(s=0)$ $\equiv \widetilde{K}_{n}(0)$. Using this procedure, ${ }^{18}$ the expressions for $\widetilde{K}_{n}(0)$ for even and odd levels of truncation are

$$
\widetilde{K}_{n}(0)=\frac{K_{1}(0) K_{3}(0) \cdots K_{n-1}(0)}{K_{0}(0) K_{2}(0) \cdots K_{n-2}(0)} \widetilde{\Psi}(0), \quad n=2,4, \ldots,
$$

and

$$
\widetilde{K}_{n}(0)=\frac{K_{0}(0) K_{2}(0) \cdots K_{n-1}(0)}{K_{1}(0) K_{3}(0) \cdots K_{n-2}(0)}\left(\frac{1}{\widetilde{\Psi}(0)}\right), \quad n=3,5, \ldots,
$$

respectively, where

$$
\widetilde{\Psi}(0)=\frac{m D}{k_{B} T} .
$$

Note that $\widetilde{K}_{1}(0)=K_{0}(0) / \widetilde{\Psi}(0)$ for $n=1$. Hence, the truncation schemes at different orders not only require a knowledge of the Mori constants which are related to the frequency moments [Eq. (15)] but also require the value of the selfdiffusivity $D$. Once $\widetilde{K}_{n}(0)$ is obtained for a given level of truncation the corresponding VACF, $\Psi_{n}(t)$, is obtained by taking the inverse Laplace transform of $\widetilde{\Psi}(s)$. Using this procedure, ${ }^{18}$ the expressions for $\Psi(t)$ at the second- and third-order truncations are given below.

\section{Second-order truncation model}

$$
\Psi_{2}(t)=\left(\cos \varpi_{2} t+\frac{\widetilde{K}_{2}(0)}{2 \varpi_{2}} \sin \varpi_{2} t\right) \exp \left(-\frac{\widetilde{K}_{2}(0) t}{2}\right),
$$

where $\varpi_{2}^{2}=K_{1}(0)-\frac{1}{4} \widetilde{K}_{2}(0)^{2}>0$ and

$$
\Psi_{2}(t)=\left(\cosh \varpi_{2} t+\frac{\widetilde{K}_{2}(0)}{2 \varpi_{2}} \sinh \varpi_{2} t\right) \exp \left(-\frac{\widetilde{K}_{2}(0) t}{2}\right),
$$

where $\varpi_{2}^{2}=\frac{1}{4} \widetilde{K}_{2}(0)^{2}-K_{1}(0)>0$. In the above expression, we have used, $K_{0}(0)=1$ and $\widetilde{K}_{2}(0)=K_{1}(0) \widetilde{\Psi}(0)$ [Eq. (17), $n=2]$.

\section{Third-order truncation model}

The continued fraction representation for the third order is

$$
\tilde{\Psi}(s)=\frac{s^{2}+\widetilde{K}_{3}(0) s+K_{2}(0)}{s^{3}+\widetilde{K}_{3}(0) s^{2}+\left(K_{2}(0)+K_{1}(0)\right) s+K_{1}(0) \widetilde{K}_{3}(0)} .
$$

Using partial fraction to represent $\widetilde{\Psi}_{3}(s)$ and taking the inverse Laplace transform

$$
\Psi_{3}(t)=a e^{s_{1} t}+b e^{s_{2} t}+c e^{s_{3} t},
$$

where $s_{1}, s_{2}, s_{3}$ are roots of the cubic polynomial in the denominator of $\widetilde{\Psi}_{3}(s)$ in Eq. (22) and $a, b, c$ are given by

$$
\begin{aligned}
& a=\frac{s_{1}^{2}+\widetilde{K}_{3}(0) s_{1}+K_{2}(0)}{\left(s_{1}-s_{2}\right)\left(s_{1}-s_{3}\right)}, \\
& b=\frac{s_{2}^{2}+\widetilde{K}_{3}(0) s_{2}+K_{2}(0)}{\left(s_{2}-s_{1}\right)\left(s_{2}-s_{3}\right)}, \\
& c=\frac{s_{3}^{2}+\widetilde{K}_{3}(0) s_{3}+K_{2}(0)}{\left(s_{3}-s_{2}\right)\left(s_{3}-s_{1}\right)},
\end{aligned}
$$

and $\widetilde{K}_{3}(0)=K_{2}(0) /\left[K_{1}(0) \widetilde{\Psi}(0)\right]$ from Eq. (18).

\section{Analytic closure scheme}

In the analytic closure schemes, the continued fraction representation given in Eq. (8) is closed at the $n$th level by 
assuming a particular form for $K_{n}(t)$. This procedure can be used to obtain analytic expressions for the self-diffusivity at different levels of closure. ${ }^{20}$ In this manuscript we consider three analytic closure models that have been proposed for a bulk fluid

$$
\begin{aligned}
& K_{n}(t)=K_{n}(0) \exp \left(-\sqrt{K_{n+1}(0)} t\right), \\
& K_{n}(t)=K_{n}(0) \exp \left(-\frac{1}{2} K_{n+1}(0) t^{2}\right),
\end{aligned}
$$

and

$$
K_{n}(t)=K_{n}(0) \operatorname{sech}\left(-\sqrt{K_{n+1}(0)} t\right) .
$$

We illustrate the derivation of the expression for selfdiffusivity for the closure given in Eq. (27). Taking the Laplace transform of Eq. (27) at $s=0$

$$
\begin{aligned}
\widetilde{K}_{n}(0) & =\int_{0}^{\infty} \exp \left(-\sqrt{K_{n+1}(0)} t\right) d t \\
& =\frac{K_{n}(0)}{\sqrt{K_{n+1}(0)}} .
\end{aligned}
$$

In a similar manner, expressions for $\widetilde{K}_{n}(0)$ from Eq. (28) and Eq. (29) are

$$
\widetilde{K}_{n}(0)=\sqrt{\frac{\pi}{2}} \frac{K_{n}(0)}{\sqrt{K_{n+1}(0)}},
$$

and

$$
\widetilde{K}_{n}(0)=\frac{\pi}{2} \frac{K_{n}(0)}{\sqrt{K_{n+1}(0)}},
$$

respectively. In order to obtain the self-diffusivity, $D$, from the analytic closure models, Eqs. (30)-(32) can be combined with Eqs. (17) and (18) for even and odd closure schemes. The predicted values of the diffusivity from the analytic closure models do not involve any fitted parameters and only require Mori constants as the input. In addition, we also evaluate the model

$$
K(t)=K_{1}(0) \exp \left(-\frac{\omega_{G} t^{2}}{2}\right),
$$

where

$$
\omega_{G}=\frac{\sqrt{\pi}}{2} \omega_{v}^{2} \frac{D}{T} .
$$

This model, commonly referred to as the Gaussian, requires the self-diffusivity as the input. In our work, we have used the memory kernels described by Eqs. (27)-(29) at the first level of closure $(n=1)$. Hence, these models require the knowledge of $K_{1}(0)$ and $K_{2}(0)$, which are related to the second and fourth frequency moments $\omega_{v}^{2}$ and $\omega_{v}^{4}$ [Eq. (15)]. In order to obtain the VACF predicted by these various heuristic forms of the memory kernel, we numerically solve the ME [Eq. (2)] by substituting expressions from Eqs. (27)(29), and (33) for $n=1$. These models are referred to as models E, G1, S, and G2, respectively, whereas the secondand third-order truncation models discussed in the previous section are referred to as $\mathrm{T} 2$ and $\mathrm{T} 3$, respectively.

\section{STRUCTURED WALL}

(a)

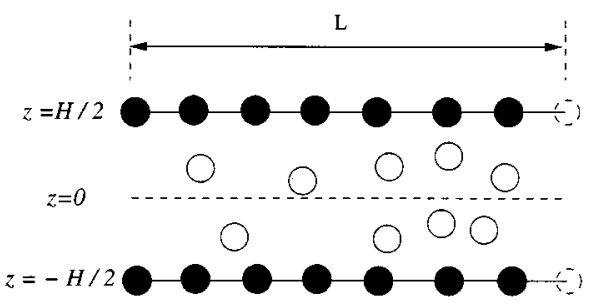

SMOOTH WALI

(b)

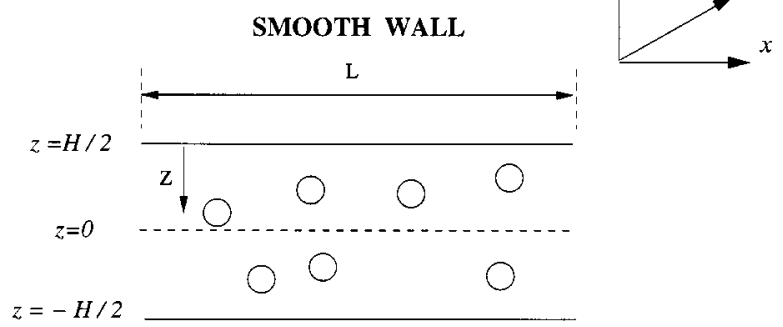

FIG. 1. Schematic of structured (a) and smooth (b) slit pore indicating the pore width $H$ and box length $L$. Periodic boundary conditions are applied in the $x$ and $y$ directions.

\section{Fluid-fluid and fluid-wall interaction potentials}

We have studied two types of slit-shaped pores. Figure 1(a) is a structured pore, where the pore wall consists of discrete particles and in Fig. 1(b), the pore wall is smooth. Both the systems are infinite in the $x-y$ plane, but confined in the $z$ direction. For a confined fluid, the total potential energy $U=U_{f f}+U_{f w}$, where $U_{f f}$ is the total fluid-fluid interaction energy and $U_{f w}$ is the total fluid-wall interaction. For a system with $N$ fluid particles with pairwise additive potentials

$$
U_{f f}=\sum_{i=1}^{N-1} \sum_{j>i} u\left(r_{i j}\right),
$$

where $u\left(r_{i j}\right)$ represents the pair potential for the particles separated by a distance $r_{i j}$. The fluid-fluid interactions for both the smooth and structured pores were assumed to be a Lennard-Jones (LJ) 12-6 potential

$$
u\left(r_{i j}\right)=4 \epsilon_{f f}\left[\left(\frac{\sigma_{f f}}{r_{i j}}\right)^{12}-\left(\frac{\sigma_{f f}}{r_{i j}}\right)^{6}\right],
$$

where $\epsilon_{f f}$ and $\sigma_{f f}$ are the LJ potential parameters. For the structured pore, fluid-wall interactions are modeled using the LJ 12-6 potential with the potential parameters $\epsilon_{f w}$ and $\sigma_{f w}$. The total fluid-wall potential for the structured pore consisting of $N_{w}$ wall particles is

$$
U_{f w}=\sum_{i=1}^{N} \sum_{j=1}^{N_{w}} u\left(r_{i j}\right) .
$$

For the smooth pore, where the fluid-wall potential is only a function of the normal distance, $z$ of the fluid particle from the wall, fluid-wall interactions were modeled using a 10-4 potential

$$
u_{f w}=2 \pi \epsilon_{f w}\left[\frac{2}{5}\left(\frac{\sigma_{f w}}{z}\right)^{10}-\left(\frac{\sigma_{f w}}{z}\right)^{4}\right] .
$$


Equation (37) represents the interaction of the fluid particle with a single layer of atoms arranged in an fcc lattice. The total fluid-wall interaction in the smooth pore is the sum of the potential energies arising from two walls located at $z_{w}$ $=-H / 2$ and $z_{w}=+H / 2$, respectively,

$$
U_{f w}=\sum_{i=1}^{N} u_{f w}\left(z_{i}+\frac{H}{2}\right)+u_{f w}\left(z_{i}-\frac{H}{2}\right),
$$

where $u_{f w}$ is given in Eq. (37) and $z_{i}$ is the $z$ coordinate of the $i$ th fluid particle.

The fluid-fluid and fluid-wall LJ interaction parameters used in this study for both the structured and smooth pores correspond to those of argon, $\sigma_{f f}=\sigma_{f w}=3.405 \AA$ and $\epsilon_{f f}$ $=\epsilon_{f w}=120 \mathrm{~K}$. In the case of the structured pore, each wall consisted of 200 particles arranged in an fcc lattice. This corresponds to a pore of width $L=14.14 \sigma$. The walls were placed such that the atoms on opposite walls were in registry.

\section{E. Evaluation of frequency moments and application to confined system}

We have modeled the VACF of a fluid confined in a slit pore based on truncation as well as analytic closure schemes discussed above. In order to evaluate the expressions for $\Psi(t)$ from the truncation and analytic closure models described above, one requires the values for the Mori constants $K_{n}(0)$ which are related to the frequency moments as given in Eq. (15). We evaluate the frequency moments from trajectories generated during the molecular dynamic simulations. From Eq. (10), the frequency moments can be directly obtained from the particle velocities, ${ }^{18}$ using

$$
\omega_{v}^{2}=\frac{\left\langle\dot{v}_{x}(0)^{2}+\dot{v}_{y}(0)^{2}+\dot{v}_{z}(0)^{2}\right\rangle_{t, N}}{\left\langle v_{x}(0)^{2}+v_{y}(0)^{2}+v_{z}(0)^{2}\right\rangle_{t, N}},
$$

where $v_{x}, v_{y}$ and $v_{z}$ are the single particle velocities in Cartesian coordinates, $\langle\ldots\rangle_{t, N}$ denotes time as well as particle averages, and

$$
\omega_{v}^{4}=\frac{\left\langle\ddot{v}_{x}(0)^{2}+\ddot{v}_{y}(0)^{2}+\ddot{v}_{z}(0)^{2}\right\rangle_{t, N}}{\left\langle v_{x}(0)^{2}+v_{y}(0)^{2}+v_{z}(0)^{2}\right\rangle_{t, N}} .
$$

As $\dot{v}(0)$ is proportional to the force experienced by a particle, Eq. (39) can be expressed using the zero time force autocorrelation function. Hence

$$
\omega_{v}^{2}=\frac{1}{m} \frac{\left\langle F_{x}(0)^{2}+F_{y}(0)^{2}+F_{z}(0)^{2}\right\rangle_{t, N}}{\left\langle v_{x}(0)^{2}+v_{y}(0)^{2}+v_{z}(0)^{2}\right\rangle_{t, N}} .
$$

Similarly, Eq. (40) is the autocorrelation between first derivative of forces; hence

$$
\omega_{v}^{4}=\frac{1}{m} \frac{\left\langle\dot{F}_{x}(0)^{2}+\dot{F}_{y}(0)^{2}+\dot{F}_{z}(0)^{2}\right\rangle_{t, N}}{\left\langle v_{x}(0)^{2}+v_{y}(0)^{2}+v_{z}(0)^{2}\right\rangle_{t, N}} .
$$

In addition, the moments can also be evaluated from the interatomic potentials. ${ }^{18,19,31}$ For a confined fluid the expressions for $\dot{v}_{x}(0)$ and $\ddot{v}_{x}(0)$ in terms of the interatomic pair potentials are
TABLE I. Reduced units used in this work. $\epsilon_{f f}$ and $\sigma_{f f}$ are the LennardJones parameters for the fluid-fluid interactions.

\begin{tabular}{ll}
\hline \hline \multicolumn{1}{c}{ Quantity } & \multicolumn{1}{c}{ Reduced unit } \\
\hline Potential energy & $u^{*}=u / \epsilon_{f f}$ \\
Slit width & $H^{*}=H / \sigma_{f f}$ \\
Box length & $L^{*}=L / \sigma_{f f}$ \\
Time & $t^{*}=t \sqrt{\epsilon_{f f} / m \sigma_{f f}^{2}}$ \\
Temperature & $T^{*}=k T / \epsilon_{f f}$ \\
Density & $\rho^{*}=\rho \sigma_{f f}^{3}$ \\
Felocity & $v^{*}=v \sqrt{m / \epsilon_{f f}}$ \\
Second frequency moment & $F^{*}=F \sigma_{f f} / \epsilon_{f f}$ \\
Fourth frequency moment & $\left(\omega_{v}^{2}\right)^{*}=\omega_{v}^{2} m \sigma_{f f}^{2} / \epsilon_{f f}$ \\
Diffusivity & $\left(\omega_{v}^{4}\right)^{*}=\omega_{v}^{4}\left(m \sigma_{f f}^{2} / \epsilon_{f f}\right)^{2}$ \\
\hline \hline & $D^{*}=D \sigma_{f f} \sqrt{\epsilon_{f f} / m}$ \\
\hline$\left.\dot{v}_{x}(0)^{2}\right\rangle_{t, N}=\frac{k_{B} T}{m^{2}}\left\langle\frac{1}{N} \sum_{i}^{N}\left[\sum_{j \neq i}^{N} \frac{\partial^{2} u\left(r_{i j}\right)}{\partial x_{i}^{2}}\right.\right.$ \\
$\left.\left.\qquad \sum_{w} \frac{\partial^{2} u\left(r_{i w}\right)}{\partial x_{i}^{2}}\right]\right\rangle$
\end{tabular}

and

$$
\begin{aligned}
\left\langle\ddot{v}_{x}(0)^{2}\right\rangle_{t, N}= & \frac{k_{B} T}{m^{3}}\left\langle\frac { 1 } { N } \sum _ { i } ^ { N } \left[\sum_{j \neq i}^{N}\left(\sum_{\alpha} \frac{\partial^{2} u\left(r_{i j}\right)}{\partial x_{i} \partial r_{i \alpha}}\right)^{2}\right.\right. \\
& +\left(\sum_{j \neq i}^{N} \sum_{\alpha} \frac{\partial^{2} u\left(r_{i j}\right)}{\partial x_{i} \partial r_{i \alpha}}\right. \\
& \left.\left.\left.+\sum_{w}^{N_{w}} \sum_{\alpha} \frac{\partial^{2} u\left(r_{i w}\right)}{\partial x_{i} \partial r_{i \alpha}}\right)^{2}\right]\right\rangle_{t},
\end{aligned}
$$

where $x_{i}$ is the $x$ position of particle $i, \alpha=x, y, z$, and $r_{i \alpha}$ is the corresponding position of particle $i$; for instance $r_{i x}$ $=x_{i}$. The expressions for the terms involving second derivatives for an $\mathrm{LJ}$ pair potential are

$$
\begin{aligned}
\frac{\partial^{2} u\left(r_{i j}\right)}{\partial x_{i}^{2}}= & -\frac{24}{r_{i j}^{2}}\left[\left(\frac{2}{r_{i j}^{12}}\right)-\left(\frac{1}{r_{i j}^{6}}\right)\right]+\left(\frac{96}{r_{i j}^{4}}\right) \\
& \times\left[\left(\frac{7}{r_{i j}^{12}}\right)-\left(\frac{2}{r_{i j}^{6}}\right)\right] x_{i j}^{2}, \\
\sum_{\alpha} \frac{\partial^{2} u\left(r_{i j}\right)}{\partial x_{i} \partial r_{i \alpha}}= & -\frac{24}{r_{i j}^{2}}\left[\left(\frac{2}{r_{i j}^{12}}\right)-\left(\frac{1}{r_{i j}^{6}}\right)\right]+\left(\frac{96}{r_{i j}^{4}}\right) \\
& \times\left[\left(\frac{7}{r_{i j}^{12}}\right)-\left(\frac{2}{r_{i j}^{6}}\right)\right]\left(x_{i j}+y_{i j}+z_{i j}\right) x_{i j} .
\end{aligned}
$$

\section{SIMULATION DETAILS}

Table I illustrates the reduced units used in this study, where all quantities are reduced with respect to the fluid LJ parameters. The pore width $H^{*}$ is the center-to-center distance between atoms on opposing walls. The pore density $\rho^{*}=N / V^{*}$, where $V^{*}$ is the pore volume based on a square simulation box of side $L^{*}$. All simulations were carried out in a constant temperature, $N V T$ ensemble, at $T^{*}=1.2$. The 
TABLE II. Results of MD simulations. $\left\langle U_{f f}^{*}\right\rangle / N$ and $\left\langle U_{f w}^{*}\right\rangle / N$ are the timeaveraged per particle total fluid-fluid and fluid-wall interaction energies.

\begin{tabular}{clll}
\hline \hline$H^{*}$ & $\rho^{*}$ & $\left\langle U_{f f}^{*}\right\rangle / N$ & $\left\langle U_{f w}^{*}\right\rangle / N$ \\
\hline 2.00 & 0.26 & -1.38603 & -7.72751 \\
& 0.3 & -1.58615 & -7.74695 \\
2.75 & 0.3 & -2.02542 & -4.30602 \\
& 0.46 & -3.00734 & -4.41334 \\
\multirow{2}{*}{4.00} & 0.5 & -3.24242 & -4.43874 \\
& 0.3 & -2.15372 & -2.767 \\
\multirow{2}{*}{4.40} & 0.5 & -3.50347 & -2.73507 \\
& 0.6 & -4.11109 & -2.79134 \\
\hline \hline
\end{tabular}

total simulation length consisted of 100000 with a time step $\Delta t^{*}=0.004(\Delta t=0.008 \mathrm{ps})$, with averages collected over the last 50000 steps. The pore widths and densities studied, and the time averages of the total per particle fluid-fluid and fluid-wall interaction energies for the structured pore, are reported in Table II. Velocities at every time step were used to calculate the VACF, with the time origins shifted every 10 time steps.

A constant temperature was maintained by using a constrained dynamics method ${ }^{27-30}$ which is reported to give the correct values of transport properties and VACFs for a bulk fluid. ${ }^{32,33}$ This constrained dynamics algorithm is based on the Gauss principle of least constraint. In order to check the accuracy of the trajectories for a confined system, we compared the VACF obtained using the NVT molecular dynamics with $N V E$ dynamics. To make an accurate comparison, $N V T$ simulations were carried out at the ensemble-averaged temperature obtained from the NVE molecular dynamics simulation. Figure 2 illustrates the comparison for the smooth pore at $H^{*}=4.0, \rho^{*}=0.5$ and $T^{*}=1.173$, where both the inplane [Fig. 2(a)] and out-of-plane [Fig. 2(b)] components of the VACF show an excellent match between the two simulations, indicating that the $N V T$ dynamics can be used for the evaluation of transport properties in a slit pore.

The frequency moments, $\omega_{v}^{2}$ and $\omega_{v}^{4}$, which are used to evaluate the Mori constants [Eq. (15)], are the only inputs required by the various models. We used three different methods to validate the accuracy of the computed moments:

(a) from velocities of particles [Eqs. (39) and (40)];

(b) from forces on individual particles [Eqs. (41) and (42)], and

(c) from the interatomic potentials [Eqs. (43) and (44)] with Eqs. (39) and (40).

The results are shown in Tables III and IV for the $x, y$, and $z$ directions separately for different pore widths and densities. These moments were averaged at every time step after equilibration, and higher derivatives of the velocities and forces were computed numerically using first-order finite difference schemes. The values of $\omega_{v}^{2}$ obtained from various methods are in close agreement; however, the differences are greater for $\omega_{v}^{4}$ as higher derivatives have to be computed. The percent differences between the various methods for $\omega_{v}^{4}$ are typically between $1 \%-1.5 \%$ for the smaller pores and less than $1 \%$ for the larger pores. The corresponding Mori
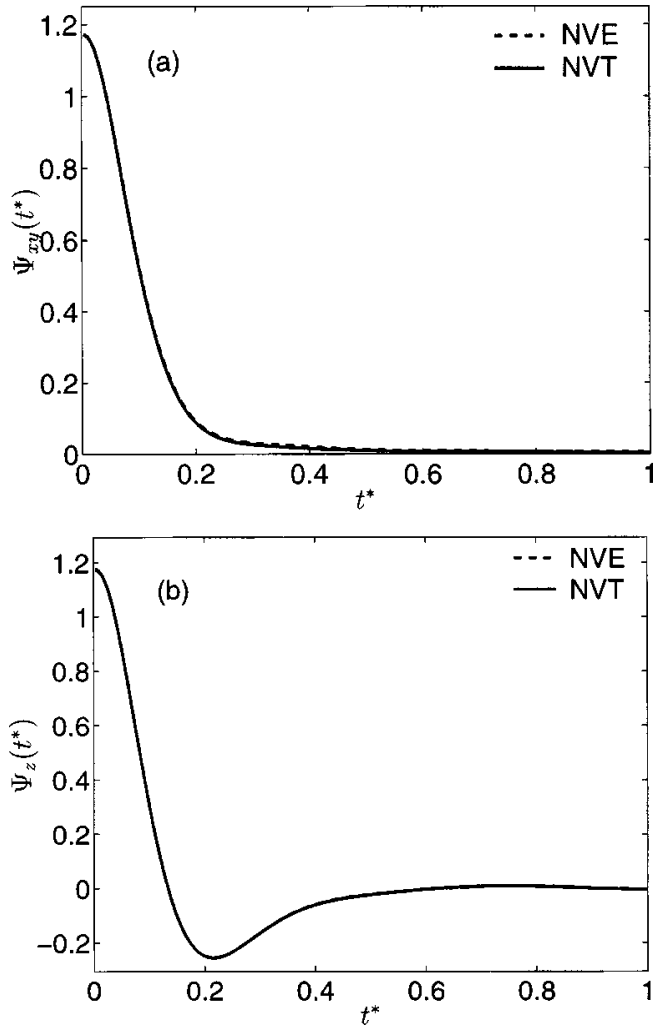

FIG. 2. Comparison of $\Psi(t)$ between $N V E$ and $N V T$ dynamics for a smooth pore. (a) $\Psi_{x y}(t)$ and (b) $\Psi_{z}(t)$ at $H^{*}=4.0, \rho^{*}=0.5$, and $T^{*}=1.173$. Both components of the VACF show an excellent match between the two simulation methods.

constants, $K_{1}(0)$ and $K_{2}(0)$, which are used in this study are computed from the frequency moments obtained from the particle velocities are shown in Table V. In order to assess the significance of the differences associated with the frequency moments (in particular for $\omega_{v}^{4}$ ) computed using the three methods, we compared the predicted VACFs for the pore system that showed the largest errors, and found that the differences in the predicted VACFs were negligible.

We also compared the Mori constants from the NVT simulations reported here, for some of the slit pores using $N V E$ molecular dynamics. The comparisons between $K_{1}(0)$ were excellent; however, deviations up to $4 \%$ were observed for $K_{2}(0)$ and up to $9 \%$ for $K_{3}(0)$, for the smaller pore widths $H^{*}=2.0$ and 2.75. The deviations for the larger pores were much smaller and within the variations observed using the different methods for computing the Mori constants. We attribute the greater differences at the smaller pore widths, in part, to the larger unbalanced forces that exist at small confinements. Perhaps larger system sizes are required to reconcile these differences. Given these differences at $H^{*}=2.0$ and $H^{*}=2.75$, we carried out a detailed comparison of the resulting VACFs, memory kernels, and predicted selfdiffusivities using the Mori constants from the two ensembles. The differences in the higher Mori constants were found to have a negligible effect on the results reported here. As an added check we also compared our values of $K_{1}(0)$ and $K_{2}(0)$ for a bulk fluid using both $N V E$ and NVT dynamics. The numbers were found to be in good agreement with 
TABLE III. Comparison of $\omega_{v}^{2}$ obtained by three different methods. All values are reported in reduced units. Values are reported for $x, y$, and $z$ components separately. $\left(\omega_{v}^{2}\right)_{x, p}^{*}$ is obtained using interatomic potentials [Eq. (43)], $\left(\omega_{v}^{2}\right)_{x, f}^{*}$ from forces [Eq. (41)], and $\left(\omega_{v}^{2}\right)_{x, v}^{*}$ from velocities of particles [Eq. (39)]. All values represent averages over particles and time. Values obtained from different methods are in excellent agreement.

\begin{tabular}{clccccccccc}
\hline \hline$H^{*}$ & $\rho^{*}$ & $\left(\omega_{v}^{2}\right)_{x, p}^{*}$ & $\left(\omega_{v}^{2}\right)_{x, f}^{*}$ & $\left(\omega_{v}^{2}\right)_{x, v}^{*}$ & $\left(\omega_{v}^{2}\right)_{y, p}^{*}$ & $\left(\omega_{v}^{2}\right)_{y, f}^{*}$ & $\left(\omega_{v}^{2}\right)_{y, v}^{*}$ & $\left(\omega_{v}^{2}\right)_{z, p}^{*}$ & $\left(\omega_{v}^{2}\right)_{z, f}^{*}$ & $\left(\omega_{v}^{2}\right)_{z, v}^{*}$ \\
\hline \multirow{2}{*}{2.00} & 0.26 & 148.5987 & 149.9674 & 149.5940 & 148.4470 & 149.3623 & 148.9779 & 235.9568 & 237.6422 & 236.6953 \\
& 0.3 & 178.2069 & 178.9186 & 178.5377 & 178.4813 & 181.4293 & 180.9082 & 229.6894 & 229.7308 & 228.9788 \\
\multirow{2}{*}{2.75} & 0.3 & 138.4253 & 139.4259 & 139.2228 & 137.3697 & 138.6366 & 138.4544 & 266.6837 & 268.1625 & 267.4573 \\
& 0.46 & 229.9128 & 229.9743 & 229.7378 & 229.7165 & 230.0492 & 229.8204 & 405.9732 & 406.2578 & 405.5503 \\
& 0.5 & 261.2795 & 262.1771 & 261.9765 & 260.4816 & 261.532 & 261.276 & 439.5713 & 439.2356 & 438.5216 \\
\multirow{2}{*}{4.00} & 0.3 & 111.9336 & 112.1904 & 112.055 & 112.0623 & 112.4776 & 112.3379 & 159.1731 & 159.8213 & 159.5487 \\
& 0.5 & 212.9814 & 213.7758 & 213.6019 & 212.6439 & 213.127 & 212.9488 & 232.3212 & 232.3421 & 232.1368 \\
& 0.6 & 313.2693 & 314.024 & 313.806 & 312.8451 & 313.9897 & 313.7705 & 282.2833 & 282.6026 & 282.4237 \\
4.40 & 0.68 & 371.2543 & 371.7052 & 371.5188 & 370.6644 & 370.9422 & 370.7576 & 438.0839 & 439.1504 & 438.8687 \\
\hline \hline
\end{tabular}

those reported in the literature, ${ }^{34}$ with our values about $1 \%$ larger for both $K_{1}$ and $K_{2}$. We note that the reported values ${ }^{34}$ are obtained from a 256 particle simulation with 2600 simulation steps and averaging carried out over 32 particles.

For all the analytical closure models $\Psi(t)$ was obtained by solving the memory equation [Eq. (2)] numerically. The ME was discretized using the trapezoidal rule with a $\Delta t^{*}$ $=0.004$. Doubling the $\Delta t^{*}$ did not alter our results. As an additional check on the method, we also recovered the memory kernel by using the predicted $\Psi(t)$ as input. ${ }^{35}$

\section{RESULTS AND DISCUSSION}

\section{A. Center-of-mass autocorrelations}

Prior to discussing the performance of various models, we first address the issue of center-of-mass motion of the fluid molecules in a structured pore. As the wall molecules are fixed, the fluid molecules in a structured pore experience a net unbalanced force, resulting in a time-dependent motion of the center of mass of the system, and the total momentum of the fluid particles is no longer conserved. Although it can be formally shown that these correlations between center of mass ${ }^{10,36,37}$ vanish in the thermodynamic limit, one needs to assess the magnitude of finite size effects on these correlations. To this end, rigorous system size checks were conducted for the smallest structured pore of width $H^{*}=2.0$ and $\rho^{*}=0.4$, where the influence of the structured wall is expected to be a maximum. Keeping all other parameters fixed, the box length was increased from $5.66 \sigma$ to $20.2 \sigma$, and the center-of-mass (COM) autocorrelation was calculated using

$$
\langle\mathbf{S}(0) \cdot \mathbf{S}(t)\rangle=\left\langle\frac{1}{N} \sum_{i}^{N} m_{i} \mathbf{v}_{i}(0) \cdot \frac{1}{N} \sum_{i}^{N} m_{i} \mathbf{v}_{i}(t)\right\rangle,
$$

where $\mathbf{S}(t)$ is the total momentum per fluid particle, $N$ is the total number of fluid particles, and $\mathbf{v}_{\mathbf{i}}$ is the velocity of the $i$ th

TABLE IV. Comparison of $\omega_{v}^{4}$ by different methods. Notation similar to Table III. $\left(\omega_{v}^{4}\right)_{x, p}^{*}$ [Eq. (44)], $\left(\omega_{v}^{4}\right)_{x, f}^{*}$ [Eq. $\left.(42)\right]$, and $\left(\omega_{v}^{4}\right)_{x, v}^{*}$ [Eq. (40)]. Even though the difference in values between different methods is higher than that for $\omega_{v}^{2}$ (Table III), the differences have a negligible influence on the predicted VACF.

\begin{tabular}{clccccccccc}
\hline \hline$H^{*}$ & $\rho^{*}$ & $\left(\omega_{v}^{4}\right)_{x, p}^{*}$ & $\left(\omega_{v}^{4}\right)_{x, f}^{*}$ & $\left(\omega_{v}^{4}\right)_{x, v}^{*}$ & $\left(\omega_{v}^{4}\right)_{y, p}^{*}$ & $\left(\omega_{v}^{4}\right)_{y, f}^{*}$ & $\left(\omega_{v}^{4}\right)_{y, v}^{*}$ & $\left(\omega_{v}^{4}\right)_{z, p}^{*}$ & $\left(\omega_{v}^{4}\right)_{z, f}^{*}$ & $\left(\omega_{v}^{4}\right)_{z, v}^{*}$ \\
\hline \multirow{2}{*}{2.00} & 0.26 & 129705.1 & 128202.8 & 130290.2 & 128214.7 & 126730.1 & 128837.5 & 104923.3 & 104985.7 & 107416.0 \\
& 0.3 & 159908.8 & 159800.2 & 162175.4 & 164291.7 & 162858.0 & 164520.6 & 103459.4 & 102750.8 & 105160.1 \\
2.75 & 0.3 & 126338.6 & 127281.0 & 128947.8 & 127434.3 & 126527.2 & 128315.9 & 227182.2 & 227606.2 & 229509.5 \\
& 0.46 & 230994.6 & 230186.5 & 232237.3 & 229152.3 & 229411.8 & 231450.0 & 388347.0 & 387926.2 & 229509.5 \\
& 0.5 & 270777.6 & 270399.9 & 272584.2 & 271442.5 & 270615.1 & 272809.0 & 430404.8 & 429200.3 & 431549.8 \\
\multirow{2}{*}{4.00} & 0.3 & 102806.9 & 101448.3 & 102344.9 & 101344.1 & 100818.1 & 101703.9 & 117392.8 & 116070.5 & 117104.8 \\
& 0.5 & 217982.6 & 217321.7 & 218641.6 & 216696.4 & 215851.6 & 216982.3 & 198242.3 & 198135.7 & 199393.0 \\
& 0.6 & 352328.8 & 353357.6 & 354798.3 & 353043.1 & 353643.1 & 355120.4 & 265149.5 & 264934.3 & 266394.9 \\
4.40 & 0.68 & 446368.1 & 447012.1 & 448532.8 & 447806.7 & 445640.5 & 447242.0 & 515603.3 & 517582.0 & 519273.3 \\
\hline \hline
\end{tabular}

TABLE V. Values of Mori constants [Eq. (15)] used for category-1 and category-2 models. Frequency moments from particle velocities. Magnitudes of second-order Mori constants are higher than the first-order constants.

\begin{tabular}{llllcccl}
\hline \hline$H^{*}$ & $\rho^{*}$ & $K_{1 x}(0)$ & $K_{1 y}(0)$ & $K_{1 z}(0)$ & $K_{2 x}(0)$ & $K_{2 y}(0)$ & $K_{2 z}(0)$ \\
\hline \multirow{2}{*}{2.00} & 0.26 & 149.5941 & 148.9779 & 236.6953 & 721.3641 & 715.8314 & 217.1203 \\
& 0.3 & 178.5377 & 180.9081 & 228.9788 & 729.8165 & 728.5068 & 230.2782 \\
2.75 & 0.3 & 139.2227 & 138.4544 & 267.4572 & 786.9753 & 788.3197 & 590.6594 \\
& 0.46 & 229.7377 & 229.8204 & 405.5503 & 781.142 & 777.2704 & 556.3804 \\
& 0.5 & 261.9765 & 261.276 & 438.5216 & 778.5145 & 782.8646 & 545.58 \\
4.00 & 0.3 & 112.055 & 112.3379 & 159.5487 & 801.2902 & 793.0015 & 574.4262 \\
& 0.5 & 213.6019 & 212.9488 & 232.1368 & 809.9919 & 805.9923 & 626.8091 \\
\multirow{2}{*}{4.40} & 0.6 & 313.806 & 313.7705 & 282.4236 & 816.8231 & 818.0137 & 660.822 \\
& 0.68 & 371.5187 & 370.7575 & 438.8687 & 835.7767 & 835.5348 & 744.3403 \\
\hline \hline
\end{tabular}



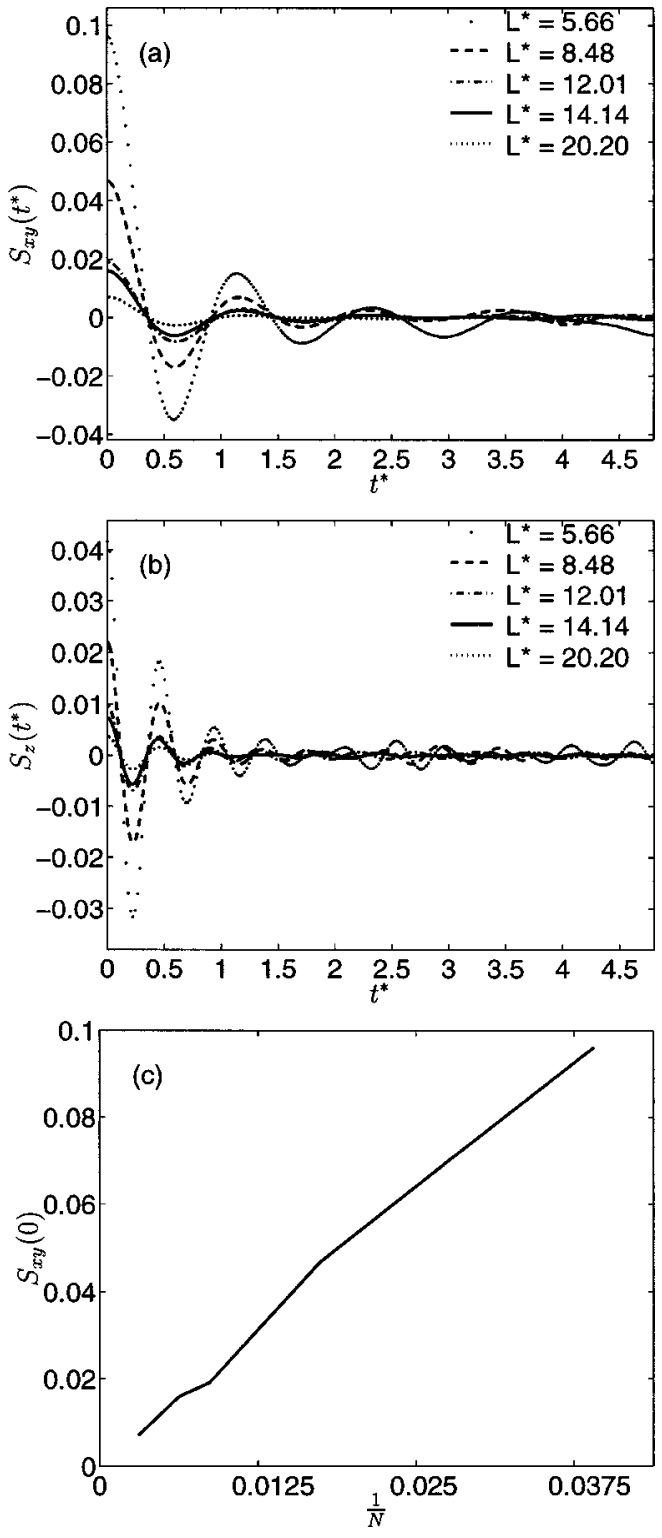

FIG. 3. Center-of-mass momentum autocorrelation as a function of box length $L^{*}$; structured pore, $H^{*}=2.0, \rho^{*}=0.4$. (a) In-plane, (b) out-of-plane momentum autocorrelation. As the system size increases, the magnitude decreases rapidly. (c) Initial value of the in-plane autocorrelation is plotted against the reciprocal of the total number of particles. A linear dependence is observed, indicating that this effect vanishes in the thermodynamic limit.

particle. The total momentum per particle was calculated at every time step after equilibration. Figures 3(a) and 3(b) illustrate the in-plane and out-of-plane correlations, respectively, for the COM autocorrelation [Eq. (47)] as a function of box length. These plots indicate that the magnitude of the COM correlations decrease as the system size is increased. From the values at $t=0$, we note that the magnitude of the COM correlations for the smallest system $(L=5.66 \sigma)$ is about an order of magnitude smaller than the kinetic temperature, $T^{*}=1.2$. A plot of initial values of the in-plane $\mathrm{COM}$ autocorrelation function against the reciprocal of the number of particles in the pore [Fig. 3(c)] reveals that the $\mathrm{COM}$ autocorrelation function scales inversely as the total number of particles. In order to investigate the influence of the COM autocorrelation on the VACF, which is the quantity
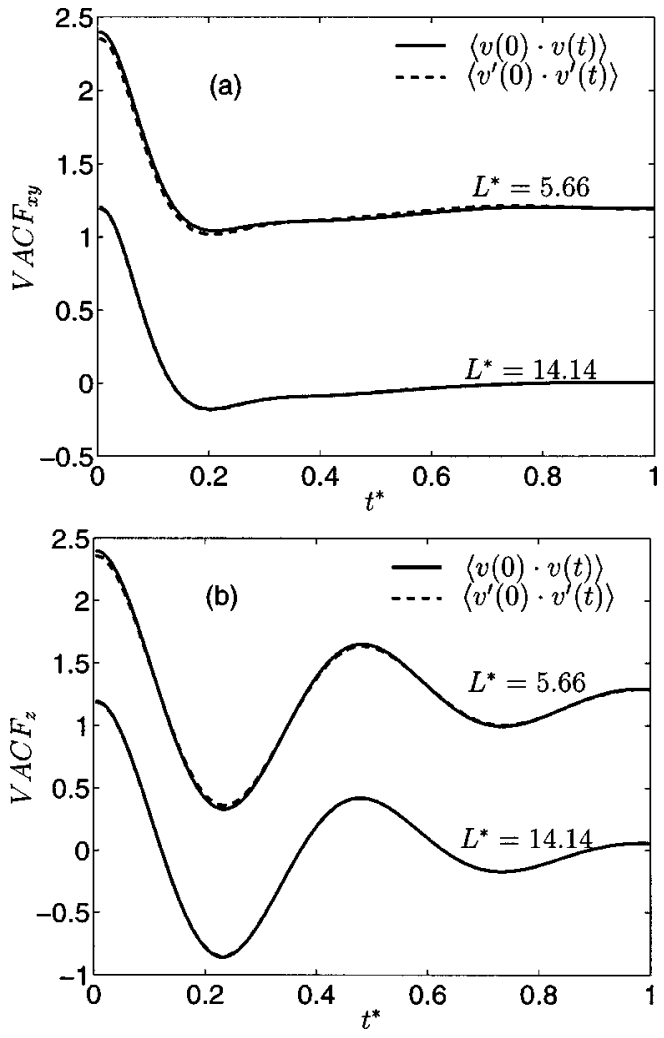

FIG. 4. Comparison between $\langle\mathbf{v}(0) \cdot \mathbf{v}(t)\rangle$ and $\left\langle\mathbf{v}^{\prime}(0) \cdot \mathbf{v}^{\prime}(t)\right\rangle$; structured pore, $H^{*}=2.0, \rho^{*}=0.4$. To obtain the latter, center-of-mass velocities were subtracted from the velocities of every particle, Eq. (48). (a) In-plane VACF, (b) out-of-plane VACF. Even for the smallest system size $\left(L^{*}=5.66\right)$ the center-of-mass motion has a small effect on the VACF. At $L^{*}=14.14$ the VACFs are indistinguishable. The ordinate for $L^{*}=5.66$ has been shifted by one unit for clarity.

of interest, we computed the VACF using the trajectories $\mathbf{v}(t)$ generated directly from the simulation with the trajectories $\mathbf{v}^{\prime}(t)$ in the COM reference frame, where

$$
\mathbf{v}^{\prime}(t)=\mathbf{v}(t)-\frac{1}{N} \sum_{i}^{N} \mathbf{v}_{i}(t)
$$

The comparison between the VACFs between the primed and unprimed velocities illustrated in Fig. $4\left(\rho^{*}=0.4, H^{*}\right.$ $=2.0$ ) indicates that even for the smallest system size ( $L$ $=5.66 \sigma)$ the COM motion has a small effect on the VACF. At $L=14.14 \sigma$ the VACFs are indistinguishable, indicating that as the system size increases the influence of the COM motion on the particle dynamics is negligible. Based on this study, all simulations reported in this manuscript were carried out at $L=14.14 \sigma$.

\section{B. Diffusive behavior in the in-plane and out-of-plane direction}

Since the slit pores represent a geometry where the system is finite in the $z$ direction, the particles are not expected to be diffusive in this direction. ${ }^{3,38}$ The mean-squared displacement in the $x-y$ and $z$ directions shown in Figs. 5(a) and 5(b), respectively, for selected pore widths and densities. The linear regimes in the $x-y$ plane are rapidly established; however, the absence of the linear regime in the $z$ direction clearly indicates the nondiffusive character in the $z$ direction. 

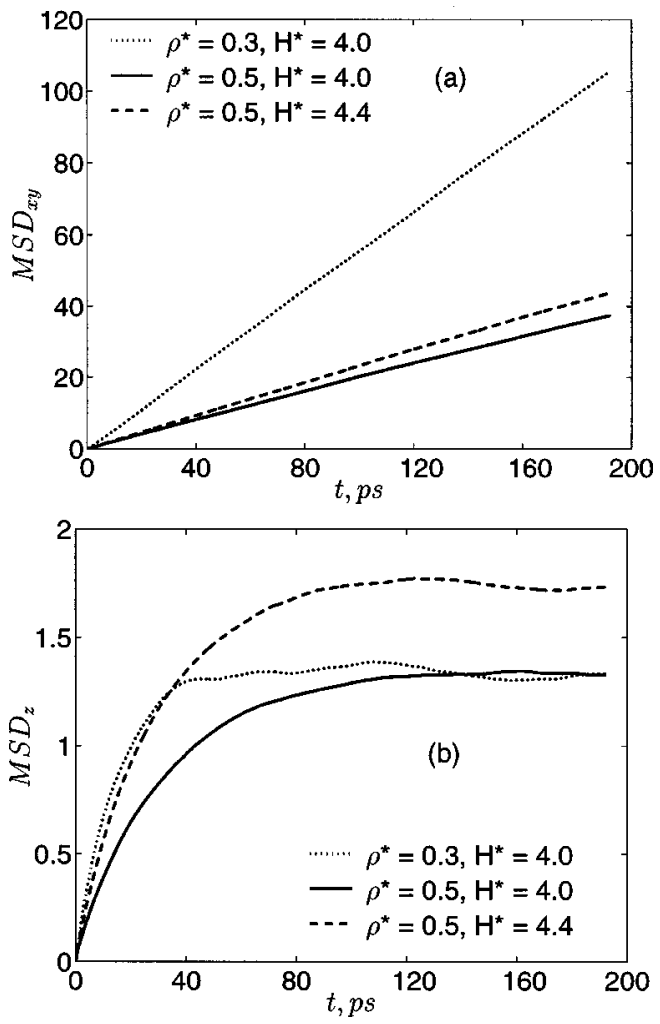

FIG. 5. Mean-square displacements (MSD) for structured pores (a) in-plane MSD is linear and the slope, which is proportional to the in-plane selfdiffusivity, is seen to increase with reduction in density and increase in pore width. (b) MSD in the $z$ direction; the absence of the linear regime clearly indicates the nondiffusive character in the $z$ direction.

The slopes at shorter times $(5-10 \mathrm{ps})$ in the mean-squared displacement in the $z$ direction have sometimes been interpreted as an indication of the mobility of the fluid. ${ }^{3,4,7}$ Since we are concerned primarily with the performance of models for predicting the VACF in slit pores, we restrict our attention to the in-plane performance of the various models. However, in the analytic closure models where the diffusivity is not required as an input we also compare the predicted forms of the VACF in the $z$ direction with the simulation results, primarily to study the prediction of the short-time out-ofplane dynamics by the models.

While comparing the predictions of various models with the VACF obtained from the simulations, the models are grouped into two categories. $\Psi(t)$ obtained from the truncation schemes denoted as T2 [Eqs. (20) and (21)] and T3 [Eq. (23)], respectively, are grouped along with the analytic closure model G2 [Eq. (33)], forming the first category. Since these three models require the self-diffusivity obtained from the $\Psi(t)$ as inputs, only their in-plane performance is analyzed. The in-plane self-diffusivities which were used in the category-1 models were computed by integrating the inplane autocorrelation function $\Psi_{x y}(t)$. These values were found to be in good agreement with the self-diffusivities obtained from the slopes of the mean-squared displacements versus time plots (Table VI). The analytic closure models G1, E, and S [Eqs. (27)-(29), respectively] which form the second category do not require any information a priori from $\Psi(t)$.
TABLE VI. In-plane diffusivities predicted by various analytic closure models $(n=1)$ compared with MD simulations. The in-plane diffusivity $D_{x y}(M D)$ is obtained by integrating the $\Psi_{x y}(t)$ from MD simulation for 9.6 ps. The values in brackets are obtained from mean-square displacements. $D_{x y}(t)$ for analytic closure models are obtained from the $\Psi_{x y}(t)$ predicted by these models in a similar manner. In general the value predicted by models $\mathrm{S}$ gives the best agreement with MD results.

\begin{tabular}{cccccc}
\hline \hline$H^{*}$ & $\rho^{*}$ & $D_{x y}^{*}(M D)$ & $D_{x y}^{*}(S)$ & $D_{x y}^{*}(E)$ & $D_{x y}^{*}(G 1)$ \\
\hline 2.00 & 0.3 & 0.07888 & 0.11826 & 0.18941 & 0.14956 \\
4.00 & 0.3 & $\begin{array}{l}0.27446 \\
(0.2744)\end{array}$ & 0.19962 & 0.32002 & 0.25258 \\
& & & & \\
& 0.5 & 0.10234 & 0.10510 & 0.16852 & 0.13299 \\
& & $(0.1006)$ & & & \\
4.40 & 0.6 & 0.05095 & 0.07223 & 0.11581 & 0.09139 \\
& & 0.03634 & 0.06185 & 0.09920 & 0.07828 \\
& & $(0.0370)$ & & & \\
\hline
\end{tabular}

\section{Model performance for VACF}

\section{One-layered system}

We first present results for the structured pores. Figure 7 illustrates the VACF for a one-layer system $\left(H^{*}=2.0, \rho^{*}\right.$ $=0.3$ ), whose density distribution is shown in Fig. 6(a). For this system the in-plane diffusivities (Table VI) indicate that the system has a very low diffusivity when compared with the higher pore widths. The in-plane VACF, $\Psi_{x y}(t)$, are compared for the category-1 and -2 models in Figs. 7(a) and $7(b)$, respectively. The $\Psi_{x y}(t)$ obtained from simulations show broad negative regions characteristic of confined
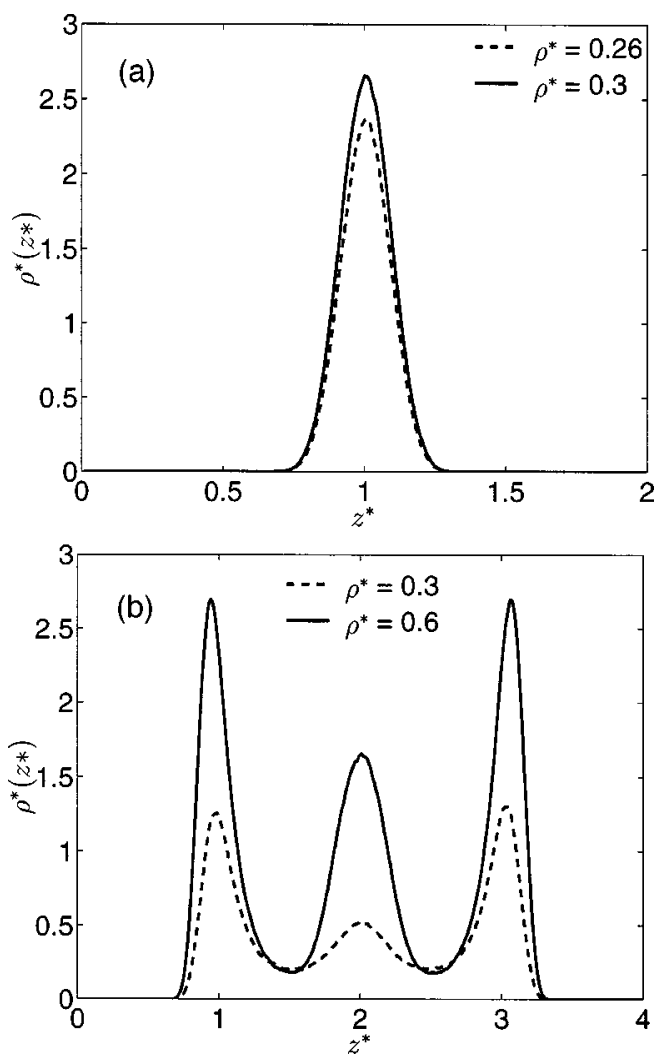

FIG. 6. Layer density distributions for structured pores. (a) $H^{*}=2.0$; onelayered system (b) $H^{*}=4.0$; three-layered system. Molecules form distinct layers upon confinement. Note that the layering is enhanced by an increase in density. 

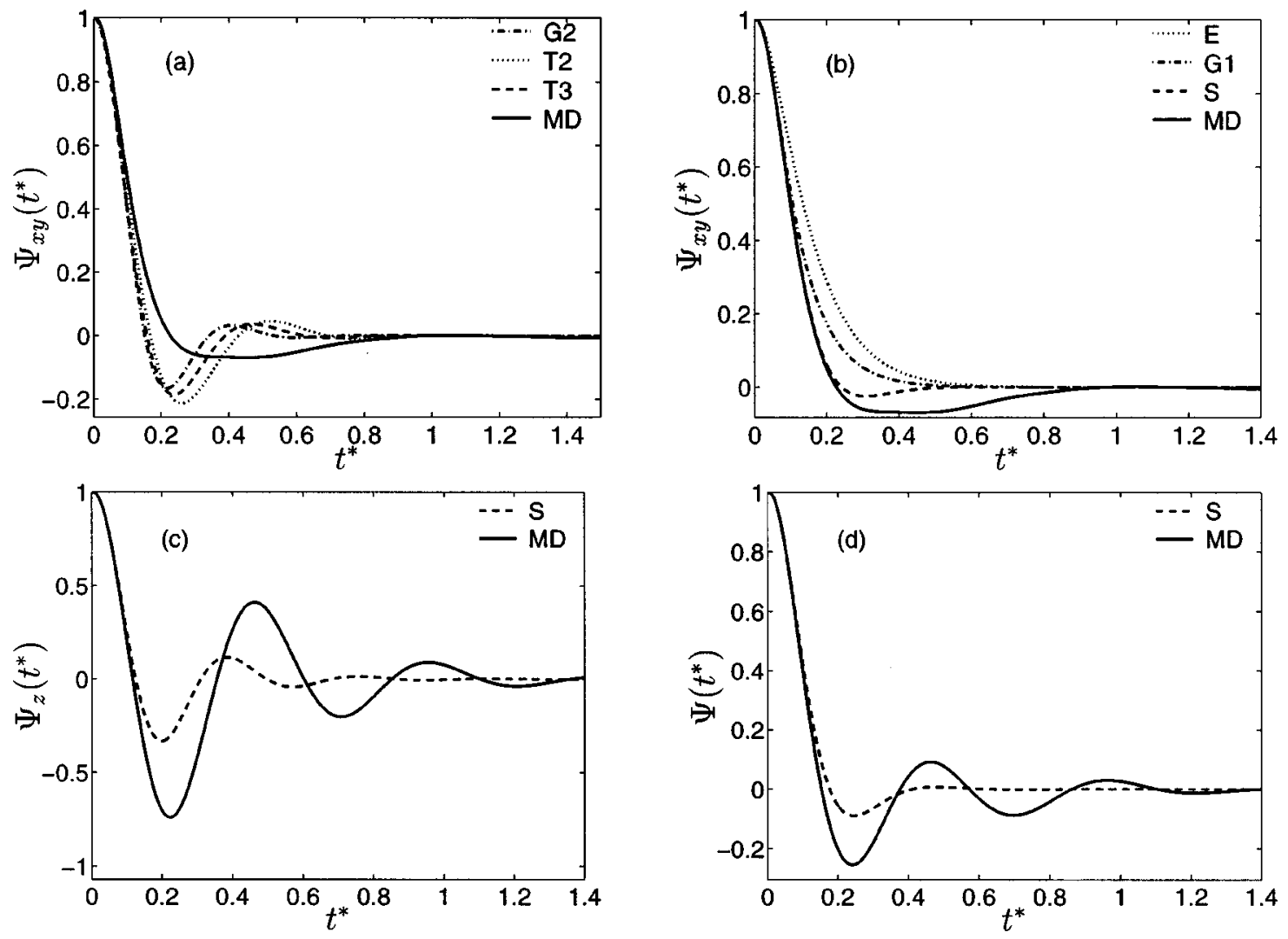

FIG. 7. Predictions of various models for a one-layer system; structured pore, $H^{*}=2.0, \rho^{*}=0.3$. The in-plane VACF, $\Psi_{x y}(t)$, obtained from simulations shows a broad negative region characteristic of confined fluids. The predictions of the category-1 models (a) in general predict a larger negative region. Among category-2 models (b), model S gives the best performance, especially at short times. (c) $\Psi_{z}(t)$ is very oscillatory due to the high confinement. (d) Comparison of model S with the overall VACF, $\Psi(t)$.

fluids. ${ }^{3,4}$ The outputs of the category-1 models in general predict a larger negative correlation than that observed in the simulations. Among the category-2 models, model S is seen to give the best performance. Except for the overestimation of negative correlations at intermediate times, the short-time behavior is captured very well. Since the performance of model $\mathrm{S}$ was the best among the category- 2 models, we compared the out-of-plane VACF, $\Psi_{z}(t)$, with that obtained from the simulations in Fig. 7(c). The magnitude of oscillations is smaller than those observed in the simulation. This is reflected in the overall VACF, $\Psi(t)$, as well [Fig. 7(d)]. For the one-layer system the fluid-wall potential has a single minima situated in the center of the pore. Hence, the motion of the particle is highly constrained in the $z$ direction, resulting in a $\Psi(t)[$ Fig. $7(\mathrm{~d})]$ which is highly oscillatory when compared with pores with increased number of layers, to be shown later. Regardless of the mismatch at intermediate times all models are seen to perform well at short times and decay to zero in a time scale similar to those observed in the simulations.

\section{Three-layered system}

As the pore width, $H^{*}$, is increased from 2.0 to 4.0 , three distinct molecular layers are formed inside the pore with density distribution, as shown in Fig. 6(b). We next discuss the results for such a three-layered system. Figure 8 illustrates the predicted VACFs for a low density situation of $\rho^{*}=0.3$. At this density the formation of layers is more diffuse when compared with the distinct layering at higher densities [Fig. 6(b)]. The $\Psi_{x y}(t)$ [Figs. 8(a) and 8(b)] are characteristic of a gas-like system due to the absence of negative correlations in the VACF. The general gas-like behavior of $\Psi_{x y}(t)$ is captured by all the category-1 models [Fig. 8(a)] with G2, T2, and T3 all performing similarly at short times, overestimating $\Psi_{x y}(t)$ at intermediate times, and tending to underestimate at later times. From the category- 2 models compared in Fig. 8(b), the performance of G1 appears to be the best, although the correlations are slightly lower than the simulated values at longer times. Model E overestimates and model S underestimates $\Psi_{x y}(t)$, relaxing very rapidly when compared with the simulated VACF. The trends in $\Psi(t)$ [Fig. $8(d)]$ are captured rather well, with the model G1 giving the best prediction.

Figure 9 illustrates VACF for an intermediate density $\rho^{*}=0.5$ where three layers form in the pore. The best predictions for $\Psi_{x y}(t)$ are observed for model S [Fig. 9(b)], where all the features are captured over the entire range. Except for a small increase in the predicted negative correlations for $0.2<t^{*}<0.3$, the prediction with model $\mathrm{S}$ is remarkably good. Although the larger negative regions in $\Psi_{z}(t)$ [Fig. 9(c)] are not adequately captured, model S appears to predict $\Psi(t)$ [Fig. 9(d)] remarkably well.

As the pore density is increased to $\rho^{*}=0.6$, the performance of the category-1 models deteriorates [Fig. 10(a)] and 

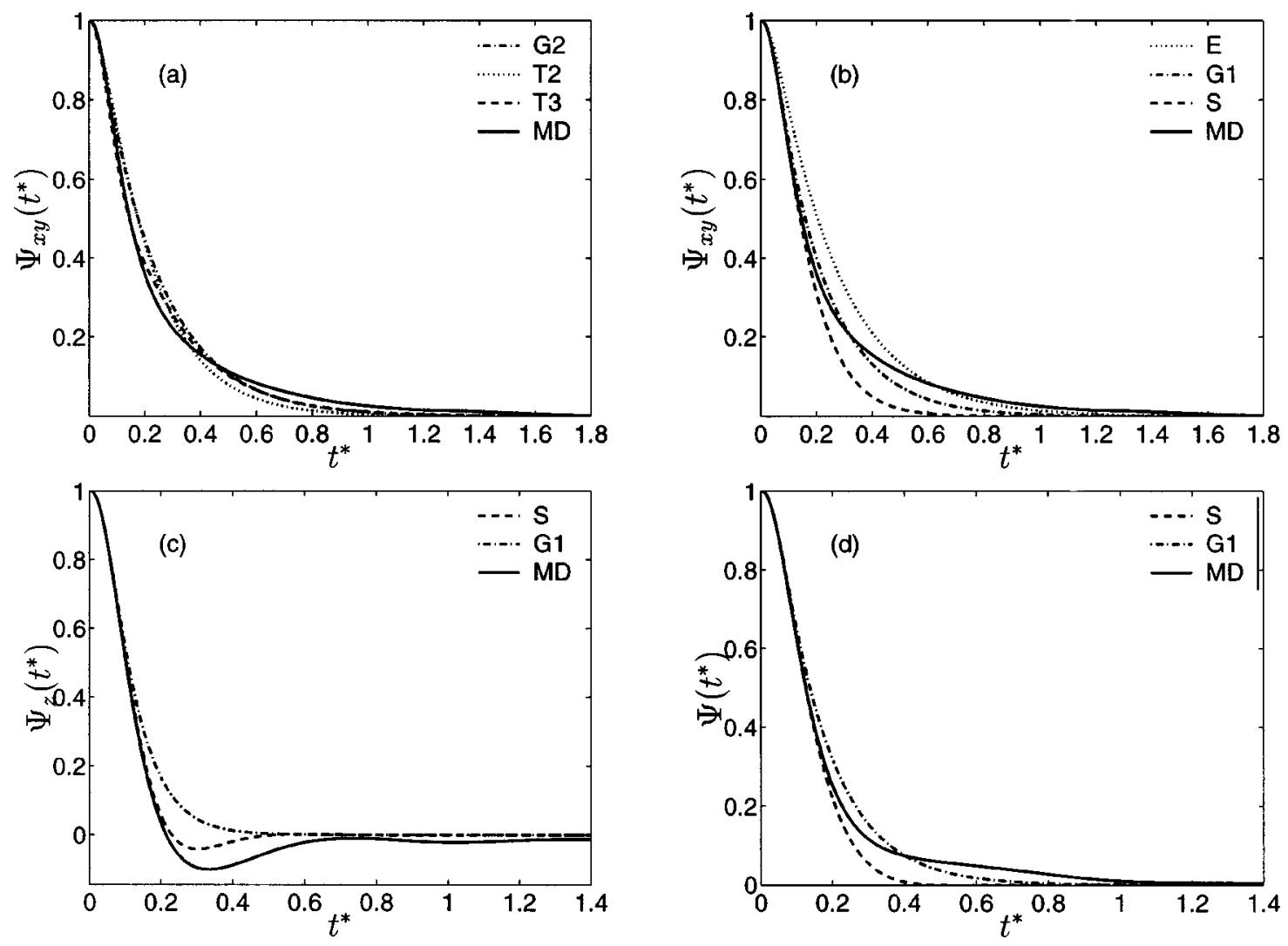

FIG. 8. Model predictions for a low density gas-like three-layer system; structured pore, $\rho^{*}=0.3, H^{*}=4.0$. Although the performance of all models is satisfactory, the predictions by model G1 (b) are closest to the simulation results. Comparison of $\Psi_{z}(t)(\mathrm{c})$ and $\Psi(t)(\mathrm{d})$ with models $\mathrm{S}$ and G1.

only model $\mathrm{S}$ [Fig. 10(b)] is able to capture $\Psi_{x y}(t)$ in a realistic manner. As observed in Fig. 10(b), both the intensity and temporal location of the negative region is predicted by the model accurately. However, model $\mathrm{S}$ fails to capture the broad negative region in $\Psi_{x y}(t)$. Category-1 models in general predict an underdamped response, a feature observed in bulk fluids, ${ }^{18,39}$ similar to their performance at smaller pore widths where only a single layer is present (Fig. 7).

We also studied a four-layered system at $H^{*}=4.4$ (Fig. 11), where the trends in the model are very similar to the highest density three-layered system investigated [Fig. 10(b)]. It is to be noticed that as the pore width increases, the match of the predictions from model $\mathrm{S}$ with simulation results improves. In particular, the improved prediction in the $z$ direction [Fig. 11(c)] results in a better prediction of the overall VACF [Fig. 11(d)] when compared with the smaller pore widths at liquid-like densities.

We have also carried out simulations for a two-layered system $\left(H^{*}=2.75, \rho^{*}=0.5\right)$. The results (not shown) are qualitatively similar to the results for the high density threelayered system (Fig. 10) with the category-1 models, G2, T2, and $\mathrm{T} 3$ predicting an oscillatory or underdamped VACF and improved performance from the category-2 models E, G1, and $\mathrm{S}$, with the model $\mathrm{S}$ providing the best description.

In order to assess the influence of the structure of the pore wall on the dynamics and performance of the various models, we carried out a few simulations using the smooth 10-4 wall potential. Results for a three-layered system are shown in Fig. 12. With the exception of an increased oscil- lation in the $z$ direction [Fig. 12(c)], the structure of the smooth pore VACF is very similar to the structured pore three-layered system shown in Fig. 10. The relative performance of both the category- 1 and -2 models is similar to that observed in the structured pore, with model S [Fig. 12(b)] having the best performance. The performance of the truncation models appear to be similar to those of the structured pore at the same density. We have also studied the predictions of various models for a smooth pore at lower densities $\left(\rho^{*}=0.46\right)$. The results (not shown) indicate that the predictions of truncation models T2 and T3 are in better agreement with simulation results than for a structured pore of the same density.

\section{Memory kernels}

Since we have approximated the memory kernels with analytic closures at the first level $(n=1)$, it is instructive to compare the actual first-order memory kernel, $K_{1}(t)$, with the analytic closure forms. The actual memory kernel, which corresponds to VACF from the MD simulation, can be obtained by numerically inverting ${ }^{35}$ the ME, Eq. (2). The numerical inversion is carried out by transforming the ME, which is a first kind Volterra operator, into a second kind Volterra operator for reasons of improved stability. ${ }^{18,35}$ Since model S is seen to give the best predictions for the VACF, we have plotted the actual in-plane and out-of-plane memory kernels against the predictions of model S [Eq. (29)] in Fig. 13. For a three-layered system at an intermediate density $\left(H^{*}=4.0, \rho^{*}=0.5\right)$, the actual in-plane memory kernel 

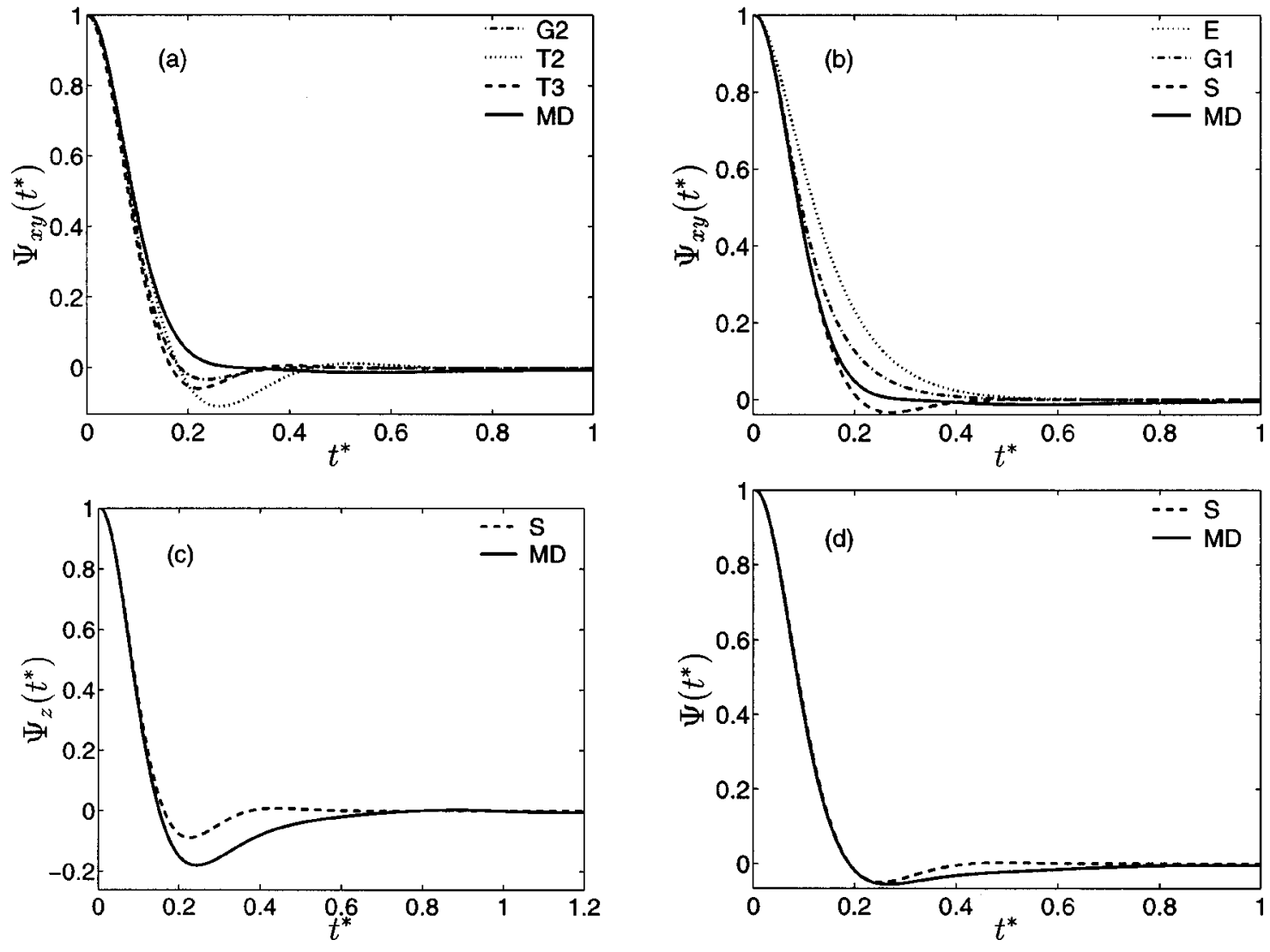

FIG. 9. Predictions for an intermediate density three-layered system; structured pore, $\rho^{*}=0.5, H^{*}=4.0$. Comparison of model $\mathrm{S}$ with MD results are very good (b). This is reflected in the overall (d) VACF as well.
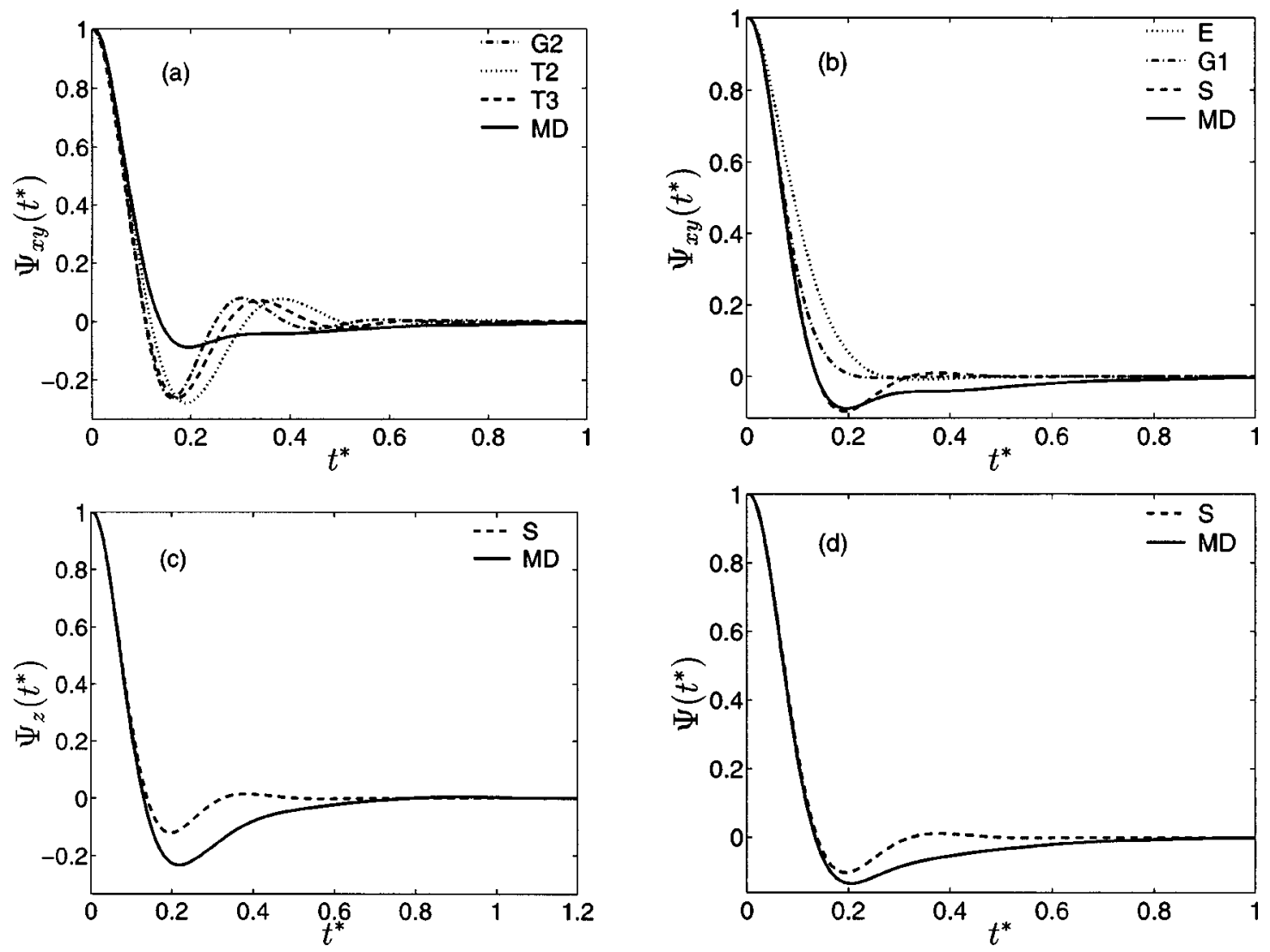

FIG. 10. Model performance for a high density three-layered system; structured pore, $\rho^{*}=0.6, H^{*}=4.0$. As the density increases, performance of category-1 models (a) deteriorates. Only model S (b) is able to capture features in $\Psi_{x y}(t)$ rather accurately. (c) and (d) show the results for $\Psi_{z}(t)$ and $\Psi(t)$, respectively. The only discrepancy is in the intermediate times, where the actual VACF relaxes much slower than the model prediction. 

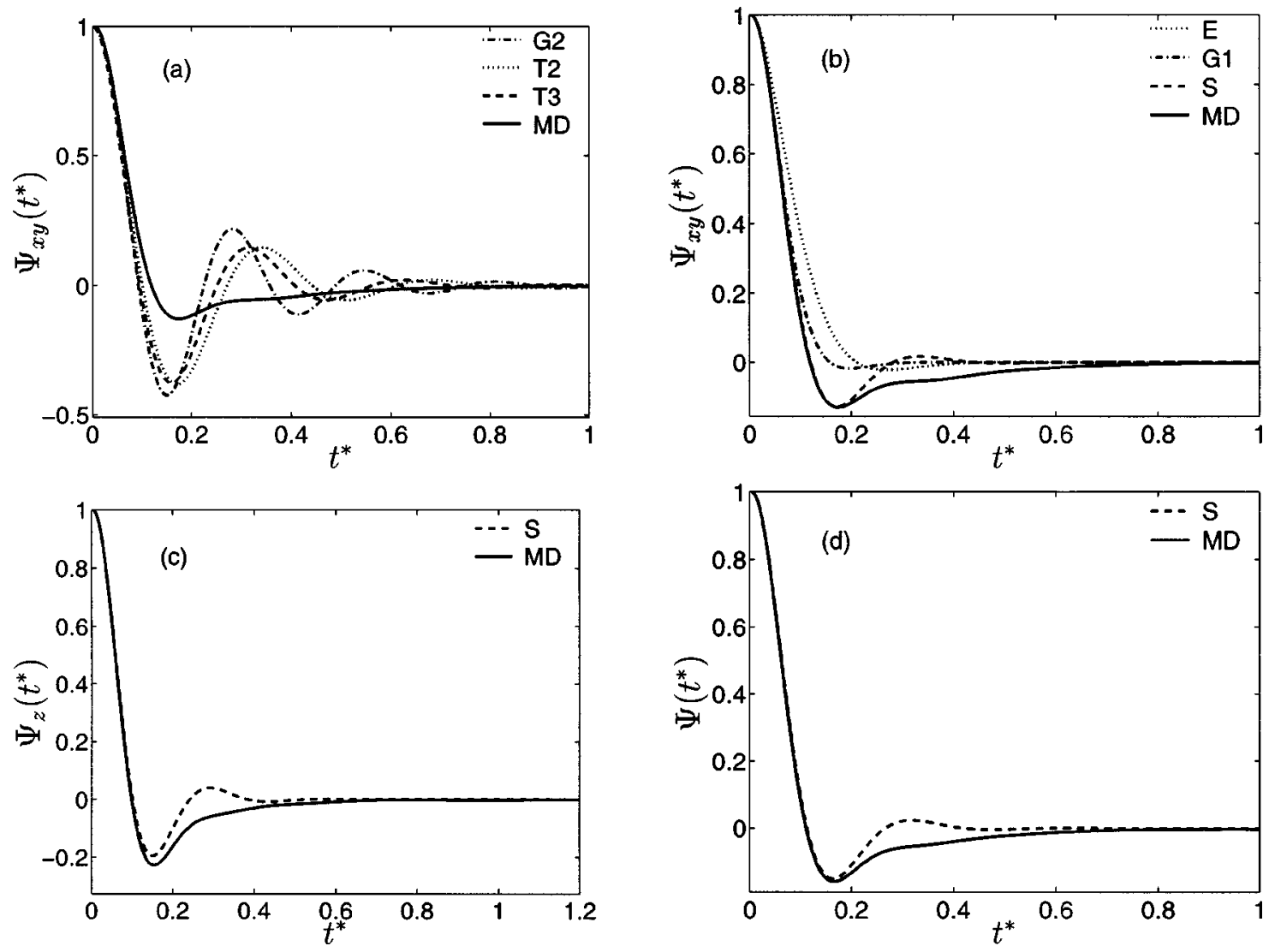

FIG. 11. Results for a four-layered system; structured pore, $H^{*}=4.40, \rho^{*}=0.68$. Performance of model S (b) at intermediate times improves in the $z$ direction as the pore width increases. This model is able to capture the minima in overall $\Psi(t)$ (d) very well.
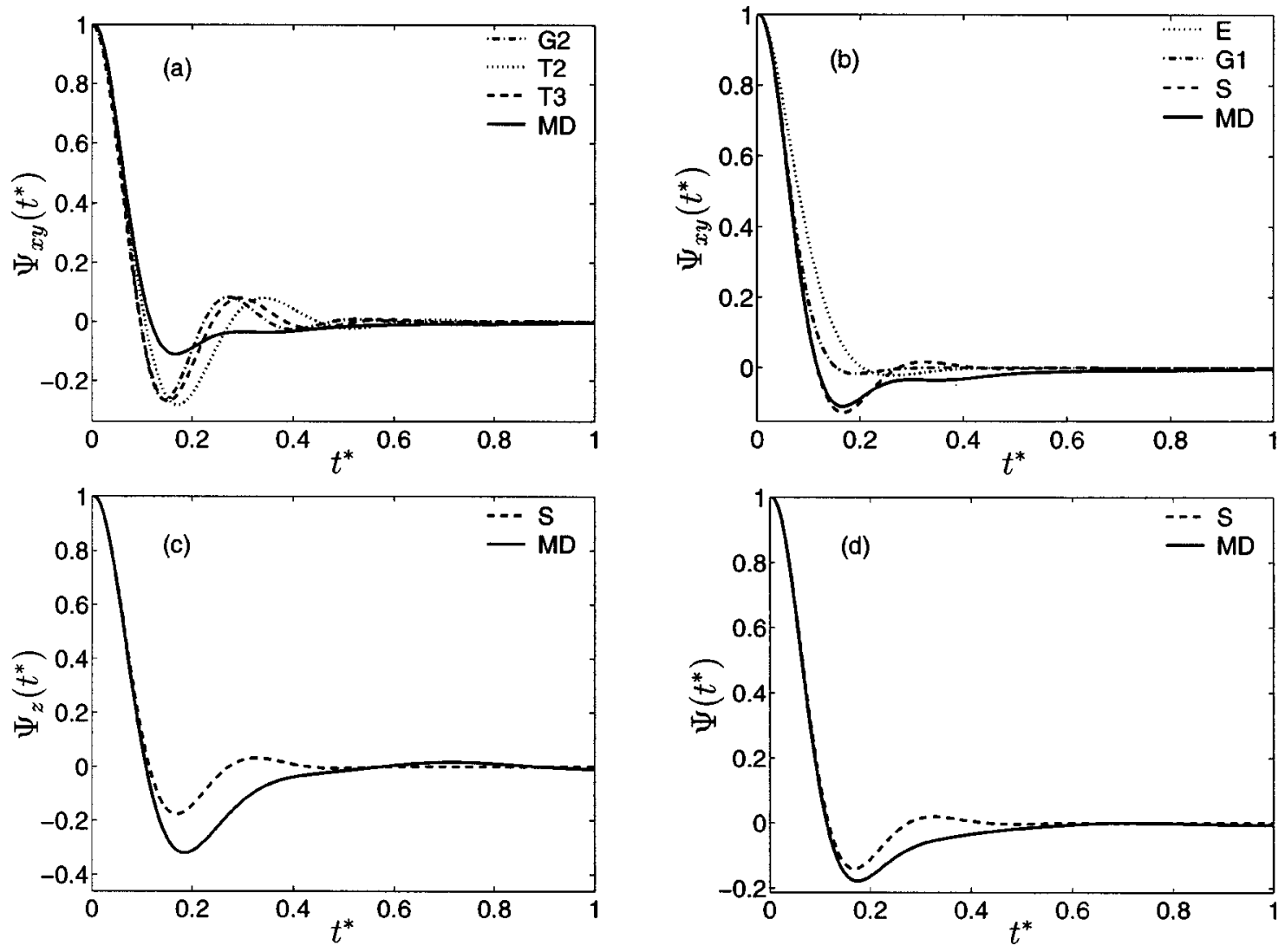

FIG. 12. Performances for a smooth pore, three-layered system $\rho^{*}=0.64, H^{*}=4.0$. Results are comparable with structured pore of same pore width, $\rho^{*}$ $=0.6$ (Fig. 10). Model S gives best results for $\Psi_{x y}(t)(\mathrm{b}), \Psi_{z}(t)(\mathrm{c})$, and $\Psi(t)(\mathrm{d})$. 

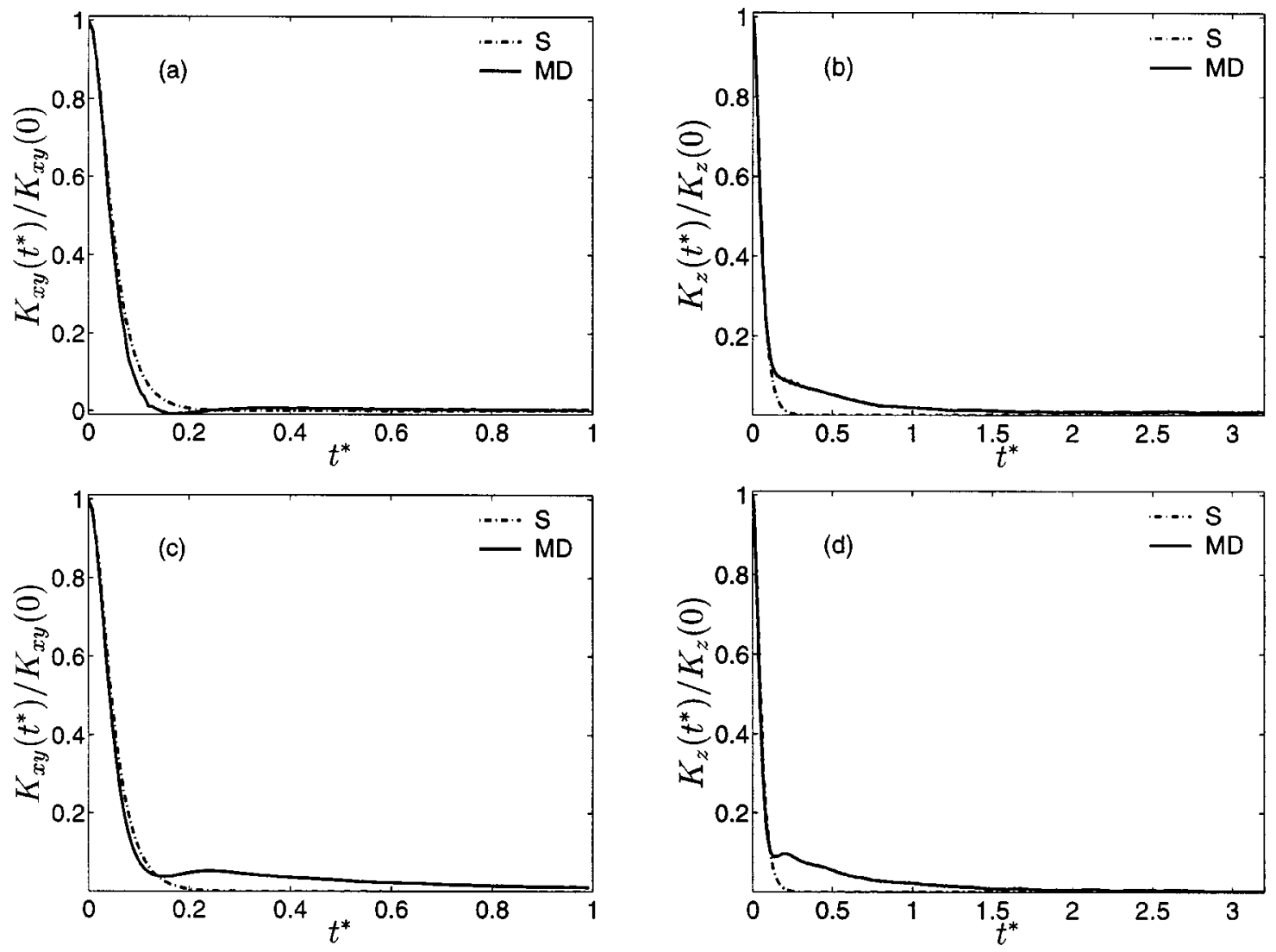

FIG. 13. Comparison between the actual normalized memory kernel obtained by solving the ME [Eq. (2)] with the simulated VACF and model S. (a) In-plane memory kernel for a three-layer system, $\rho^{*}=0.5, H^{*}=4.00$. The good agreement between the two is reflected in the $\Psi_{x y}(t)$ as well (Fig. 9). (b) Comparison for the out-of-plane memory kernel where the slower relaxation in the actual kernel is not captured by the model. (c) and (d) are the results for a four-layer system, $\rho^{*}=0.68, H^{*}=4.40$. Model is unable to capture the slow decay at intermediate times.

matches very well with the model, as shown in Fig. 13(a). The good agreement between the two is reflected in the predictions for $\Psi_{x y}(t)$ as well (Fig. 9). The in-plane memory kernel for a four-layered system $\left.\left(H^{*}=4.4,\right) \rho^{*}=0.68\right)$ has a slower decay than that predicted by the model [Fig. 13(c)]. This is reflected in the corresponding $\Psi_{x y}(t)$ [Fig. 11(b)], where the broad negative region in the VACF is not captured by the model. Comparison of out-of-plane memory kernels [Figs. 13(b) and 13(d)] show that the actual memory kernels in the $z$ direction decay much slower than their in-plane counterparts. For intermediate densities and high densities, this delay in the decay of the out-of-plane memory kernel is not captured by model $\mathrm{S}$.

We note that the negative plateau regions observed in the VACFs are due primarily to backscattering of atoms and the memory effects associated with this process. This phenomenon is also seen in bulk fluids at high densities. ${ }^{40}$ Models for the memory kernel that attempt to capture this aspect of the VACF typically involve two time constants, ${ }^{18}$ one for the short-time decay which is dominated by binary collisions and the second time constant for the slower decay arising from collective modes which are responsible for the prolonged negative correlations, ${ }^{19}$ the latter effect being more pronounced at higher densities. Under confinement this plateau region is further enhanced due to the lower dimensionality of the system. Physically, one can view this as a reduced opportunity for the particle to lose memory of it previous history, when restricted to fewer dimensions. The effect is predominant in a pure 2D system of hard disks, giving rise to the long-time tails in the VACF.

\section{E. Diffusion coefficients}

Since the category-2 models do not require a knowledge of the diffusion coefficient as inputs, they are indicative of the utility of the model in predicting the in-plane diffusivities in these systems. Time-dependent diffusivity, obtained as an integral of $\Psi_{x y}(t)$ for the category-2, analytic closure models $(n=1)$, are shown in Fig. 14. Since we are interested in the long-time limits of the integrals of the VACF, we have not used the appropriate short-time forms for the time integrals. ${ }^{18}$ The corresponding in-plane diffusion coefficients obtained by integrating the predicted VACF upto $9.6 \mathrm{ps}$ are given in Table VI.

For the smallest pore width [Fig. 14(a)] all the models overestimate the self-diffusivity. With one exception [Fig. 14(b)] model $S$ gives the best prediction for the in-plane diffusivity in the system. The best performance occurs for model $\mathrm{S}$ at intermediate densities $\left(\rho^{*}=0.5\right)$ in the threelayered system at $H^{*}=4.0$ [Fig. 14(c)]. In all models, the magnitude of the self-diffusivity is the least for model $\mathrm{S}$ and the greatest for model E (Table VI). Although there are differences between the predicted and actual diffusion coefficients, we note that at intermediate times, the model captures 

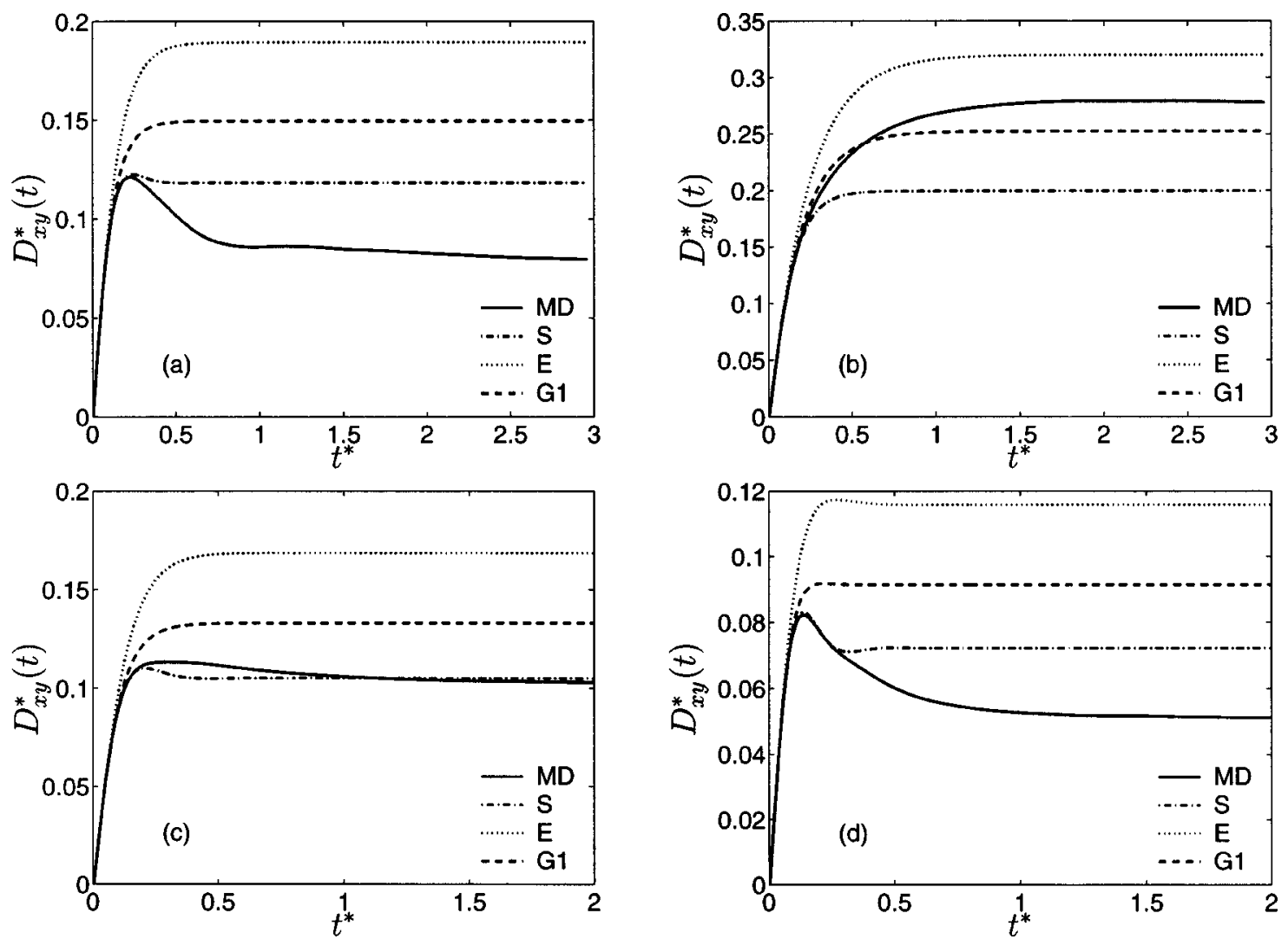

FIG. 14. In-plane time-dependent diffusivities obtained by integrating $\Psi_{x y}(t)$ from the simulations (MD) are compared with the VACFs predicted by the analytic closure models $(n=1)$ for the structured pore. (a) A one-layer system, $\rho^{*}=0.3, H^{*}=2.0$. (b) A low density three-layer system $\rho^{*}=0.3, H^{*}=4.0$. (c) An intermediate density three-layer system $\rho^{*}=0.5, H^{*}=4.0$. (d) A liquid-like system, $\rho^{*}=0.6, H^{*}=4.0$. Model $\mathrm{S}$ is closest to the simulation results with the exception of (b), where model G1 is better. Model S predicts the least value of in-plane diffusivity.

the time-dependent diffusivity very well. This situation can be observed in Figs. 14(a) and 14(d), where the corresponding VACFs are liquid-like (Figs. 7 and 10).

The in-plane self-diffusivities using the category-2 models at different levels of closure are given in Table VII. ${ }^{20}$ The

TABLE VII. In-plane diffusivities predicted by the analytic closure models at different levels of closure compared with MD results. These values are obtained without any fitted parameters or any information from MD simulation. Prediction of model S at an odd level (at higher densities) and of model $\mathrm{E}$ at an even level of truncation is in better agreement with the MD results.

\begin{tabular}{ccccccc}
\hline \hline$H^{*}$ & $\rho^{*}$ & $n$ & $D_{x y}^{*}(M D)$ & $D_{x y}^{*}(S)$ & $D_{x y}^{*}(E)$ & $D_{x y}^{*}(G 1)$ \\
\hline 2.00 & 0.3 & 0 & & 0.12836 & 0.08171 & 0.10241 \\
& & 1 & 0.07888 & 0.11479 & 0.18031 & 0.14386 \\
4.00 & 0.3 & 0 & & 0.10784 & 0.06865 & 0.08604 \\
& & 1 & 0.27446 & 0.16258 & 0.10350 & 0.12972 \\
& & 2 & & 0.20286 & 0.12914 & 0.16186 \\
& 0.5 & 0 & & 0.11795 & 0.07509 & 0.09411 \\
& & 1 & 0.10234 & 0.10176 & 0.15984 & 0.12753 \\
& & 2 & & 0.10056 & 0.06401 & 0.08023 \\
& 0.6 & 0 & & 0.09714 & 0.06184 & 0.07751 \\
& & 1 & 0.05095 & 0.06961 & 0.10934 & 0.08724 \\
4.40 & 0.68 & 0 & & 0.06488 & 0.04131 & 0.05177 \\
& & 1 & 0.03634 & 0.08933 & 0.05687 & 0.07127 \\
& & 2 & & 0.05528 & 0.09353 & 0.07463 \\
& & & & & 0.03519 & 0.04411 \\
\hline \hline
\end{tabular}

diffusion coefficients are obtained using Eqs. (17) and (18), along with Eqs. (30)-(32) for the analytic closure with $n$ $=0,1$, and 2 . Note that the diffusivities at $n=1$ compare well with the values obtained by directly integrating the predicted VACFs (Table VI), since these are in principle equivalent. We attribute the small differences due to numerical integration of the VACFs. Some general observations can be made based on the predictions of the self-diffusivities. The values of diffusivities predicted by model $\mathrm{S}$ for closure at odd $n$ at the higher densities, $\rho^{*}=0.5$ and 0.6 , are in good agreement with the MD results. The performance of model S deteriorates at lower densities as expected. Predictions for model $\mathrm{E}$ and $\mathrm{G} 1$ at even $n$ are in better agreement with the $\mathrm{MD}$ results in general. The strong dependence on the level of truncation is not so profound for model S, as is the case with the other models.

\section{SUMMARY AND CONCLUSIONS}

A good model for the velocity autocorrelation function can be used not only to predict self-diffusivities, but can also be used to understand the short-time dynamics of molecules. The VACF of a fluid confined in a slit pore exhibits either gas-like or liquid-like behavior depending on the pore densities. The time evolution equation of VACF is expressed in terms of the memory kernel. A formal solution procedure for the memory kernel involves a representation as a continued fraction and truncation at various levels. This method re- 
quires, a priori, the diffusivities as input. An alternative approach based on various analytical closure forms for the memory kernel does not require diffusivities. In a confined system, the molecules do not undergo diffusive motion in the direction normal to the wall; hence, we have modeled the in-plane VACF. From our studies, for all pore widths and densities corresponding to liquid-like VACFs, we see that the truncation models predict an oscillatory behavior for the inplane VACF. The match with the MD results is better at lower densities. All the analytical closure models were used at the first level of closure $(n=1)$ while predicting the VACF. However, in-plane diffusivities were obtained for $n$ $=0,1$, and 2. Among the analytical closure models we observe that sech is not only able to capture the short-time dynamics very well but is also seen to give the best predictions to the in-plane diffusivities at liquid-like pore densities. In a specific case of an intermediate pore density $\left(H^{*}\right.$ $=4.0, \rho^{*}=0.5, T^{*}=1.2$ ), the agreement of the prediction of sech with the simulations is excellent for the in-plane diffusivity. In this case the memory kernels also are in good agreement. In general at liquid-like densities we observe that the minima in the VACF is captured very accurately by the sech model; however, the model is unable to predict the subsequent plateau regions in the VACF. Predictions of the diffusivities appear to improve for closures at $n=0$ and 2 for the exponential model. At larger pore widths the sech model is seen to capture features in both the in-plane and out-ofplane VACFs. At the lower pore density investigated in this work, where the VACFs are gas-like, the Gaussian form of the closure gives the best performance.

Our study indicates that the analytic closure models are able to capture most of the features in confined fluid VACF. Although limited to a few densities, this study reveals that the diffusivities can sometimes be predicted with sufficient accuracy. Similar to the improvements in the theory for high density bulk fluids, any improvements in the analytic closure formalism should attempt to capture the sustained negative correlations in the VACF which are observed at intermediate times for the confined fluid.

\section{ACKNOWLEDGMENT}

The authors would like to acknowledge Biman Bagchi for useful suggestions while this work was in progress.
${ }^{1}$ H. T. Davis, Statistical Mechanics of Phases, Interfaces and Thin Films (VCH, New York, 1995).

${ }^{2}$ J. N. Israelachvili, Intermolecular and Surface Forces: With Applications to Colloidal and Bioligical Systems (Academic, London, 1985).

${ }^{3}$ J. J. Magda, M. Tirrell, and H. T. Davis, J. Chem. Phys. 83, 1888 (1985).

${ }^{4}$ M. Schoen, J. H. Cushman, D. J. Diestler, and J. C. L. Rhykerd, J. Chem. Phys. 88, 1394 (1988).

${ }^{5}$ S. A. Somers and H. T. Davis, J. Chem. Phys. 96, 5389 (1992).

${ }^{6}$ P. Bordarier, B. Rousseau, and A. H. Fuchs, Mol. Simul. 17, 199 (1996).

${ }^{7}$ M. Schoen, Computer Simulation of Condensed Phases in Complex Geometries (Springer, Berlin, 1993).

${ }^{8}$ H. T. Davis, J. Chem. Phys. 86, 1474 (1987).

${ }^{9}$ T. K. Vanderlick and H. T. Davis, J. Chem. Phys. 87, 1791 (1987).

${ }^{10}$ J. M. D. Macelroy and S. H. Suh, Mol. Simul. 2, 313 (1989).

${ }^{11}$ L. Pozhar and K. Gubbins, J. Chem. Phys. 94, 1367 (1991).

${ }^{12}$ L. Pozhar and K. Gubbins, J. Chem. Phys. 99, 8970 (1993).

${ }^{13}$ L. Pozhar and K. Gubbins, Phys. Rev. E 99, 5367 (1997).

${ }^{14}$ E. Akhmatskaya, B. Todd, P. Davis, D. J. Evans, K. Gubbins, and L. Pozhar, J. Chem. Phys. 106, 4684 (1997).

${ }^{15}$ L. Pozhar, Phys. Rev. E 61, 1432 (2000).

${ }^{16}$ W. Sung and J. Dahler, J. Chem. Phys. 80, 3025 (1984).

${ }^{17}$ M. P. Allen and D. J. Tildesley, Computer Simulation of Liquids (Clarendon, Oxford, 1987).

${ }^{18}$ J. Boon and S. Yip, Molecular Hydrodynamics (Dover, New York, 1991).

${ }^{19} \mathrm{U}$. Balucani and M. Zoppi, Dynamics of the Liquid State (Clarendon, Oxford, 1994).

${ }^{20}$ D. M. Heyes and J. G. Powles, Mol. Phys. 71, 781 (1990).

${ }^{21}$ C. G. Joslin and C. G. Gray, Mol. Phys. 58, 789 (1986).

${ }^{22}$ J. W. Allen and D. J. Diestler, J. Chem. Phys. 73, 4597 (1980).

${ }^{23}$ K. Tankeshwar, K. N. Pathak, and S. Ranganathan, J. Phys. C 20, 5749 (1987).

${ }^{24}$ K. Tankeshwar and K. N. Pathak, J. Phys.: Condens. Matter 6, 591 (1994).

${ }^{25}$ K. Tankeshwar and K. N. Pathak, J. Phys.: Condens. Matter 7, 5729 (1995)

${ }^{26}$ G. R. Kneller and K. Hinsen, J. Chem. Phys. 115, 11097 (2001).

${ }^{27}$ D. J. Evans and G. P. Morriss, Chem. Phys. 77, 63 (1983).

${ }^{28}$ D. J. Evans and G. P. Morriss, Phys. Lett. 98A, 433 (1983).

${ }^{29}$ D. J. Evans and G. P. Morriss, Comput. Phys. Rep. 1, 297 (1984).

${ }^{30}$ D. C. Rapaport, The Art of Molecular Dynamics Simulation (Cambridge University Press, Cambridge, 1998).

${ }^{31}$ B. R. A. Nijboer and A. Rahman, Physica (Amsterdam) 32, 415 (1966).

${ }^{32}$ D. Brown and J. H. R. Clarke, Mol. Phys. 51, 1243 (1984).

${ }^{33}$ J. M. Haile and S. Gupta, J. Chem. Phys. 79, 3067 (1983).

${ }^{34}$ L. L. Lee and T.-H. Chung, J. Chem. Phys. 77, 4650 (1982).

${ }^{35}$ B. J. Berne and G. Harpe, Adv. Chem. Phys. 17, 63 (1970).

${ }^{36}$ S. H. Suh and J. M. D. Macelroy, Mol. Phys. 2, 313 (1989).

${ }^{37}$ C. R. Kamala, K. G. Ayappa, and S. Y. Yashonath, Phys. Rev. E 65, 061202 (2002).

${ }^{38}$ J. G. Powles, A. Dornford-Smith, and W. A. B. Evans, Phys. Rev. Lett. 66, 1177 (1991)

${ }^{39}$ B. J. Berne, J. Boon, and S. A. Rice, J. Chem. Phys. 45, 1086 (1966).

${ }^{40}$ A. Rahman, Phys. Rev. 136, A405 (1964). 